\title{
NNLL soft and Coulomb resummation for squark and gluino production at the LHC
}

\author{
M. Beneke, ${ }^{a}$ J. Piclum, ${ }^{b, c}$ C. Schwinn ${ }^{d}$ and C. Wever ${ }^{e, f, g}$ \\ ${ }^{a}$ Physik Department T31, Technische Universität München, \\ James-Franck-Straße 1, D-85748 Garching, Germany \\ ${ }^{b}$ Theoretische Physik 1, Naturwissenschaftlich-Technische Fakultät, Universität Siegen, \\ Walter-Flex-Straße 3, D-57068 Siegen, Germany \\ ${ }^{c}$ Albert Einstein Center for Fundamental Physics, Institute for Theoretical Physics, \\ University of Bern, Sidlerstrasse 5, CH-3012 Bern, Switzerland \\ ${ }^{d}$ Institut für Theoretische Teilchenphysik und Kosmologie, RWTH Aachen University, \\ Sommerfeldstraße 16, D-52056 Aachen, Germany \\ "Institute of Nuclear Physics, NCSR "Demokritos", \\ Patriarchou Gregoriou E. \& Neapoleos street 27, GR 15310 Agia Paraskevi, Greece \\ ${ }^{f}$ Institute for Theoretical Particle Physics (TTP), Karlsruhe Institute of Technology, \\ Engesserstraße 7, D-76128 Karlsruhe, Germany \\ ${ }^{g}$ Institute for Nuclear Physics (IKP), Karlsruhe Institute of Technology, \\ Hermann-von-Helmholtz-Platz 1, D-76344 Eggenstein-Leopoldshafen, Germany \\ E-mail: piclum@physik.uni-siegen.de, schwinn@physik.rwth-aachen.de, \\ christopher.wever@kit.edu
}

ABSTRACT: We present predictions for the total cross sections for pair production of squarks and gluinos at the LHC including a combined NNLL resummation of soft and Coulomb gluon effects. We derive all terms in the NNLO cross section that are enhanced near the production threshold, which include contributions from spin-dependent potentials and socalled annihilation corrections. The NNLL corrections at $\sqrt{s}=13 \mathrm{TeV}$ range from up to $20 \%$ for squark-squark production to $90 \%$ for gluino pair production relative to the NLO results and reduce the theoretical uncertainties of the perturbative calculation to the $10 \%$ level. Grid files with our numerical results are publicly available [1].

KeYwords: NLO Computations, Supersymmetry Phenomenology

ARXIV EPRINT: 1607.07574 


\section{Contents}

1 Introduction $\quad 1$

2 NNLL soft-Coulomb resummation for squark and gluino production 4

2.1 Production processes 4

2.2 Resummation formula 6

$\begin{array}{lll}2.2 .1 & \text { Hard functions } & 7\end{array}$

2.3 Potential effects 8

2.3.1 Coulomb and non-Coulomb potential terms 11

$\begin{array}{ll}2.3 .2 & \text { Annihilation contributions } \\ & 12\end{array}$

$\begin{array}{lll}2.3 .3 & \text { NLO potential function } & 17\end{array}$

$\begin{array}{lll}2.3 .4 & \text { Bound-state effects } & 17\end{array}$

$\begin{array}{lll}\text { 2.3.5 } & \text { Fixed-order treatment of Coulomb corrections } & 18\end{array}$

2.3.6 Numerical size of non-Coulomb potential, annihilation and bound $\begin{array}{ll}\text { state contributions } & 19\end{array}$

$\begin{array}{lll}2.4 & \text { Scale choices } & 19\end{array}$

3 Numerical results $\quad 22$

$\begin{array}{lll}3.1 & \text { Setup } & 22\end{array}$

3.2 Results 24

4 Conclusions $\quad 33$

$\begin{array}{lr}\text { A Explicit formulae } & \mathbf{3 4}\end{array}$

A.1 Expansion of the NNLL cross section 34

A.2 Analytic NNLL result for fixed-order Coulomb corrections 36

\section{Introduction}

Supersymmetry (SUSY) and its realization in the $R$-parity conserving Minimally Supersymmetric Standard Model (MSSM) is a well-studied and motivated extension of the Standard Model (SM) of particle physics. It could provide a solution to shortcomings of the SM such as the absence of a dark matter candidate and it might stabilize the electroweak scale against quantum corrections. The search for SUSY at the TeV scale is therefore a central part of the physics program of the Large Hadron Collider (LHC). The production of squarks $\tilde{q}$ and gluinos $\tilde{g}$, the super-partners of quarks and gluons, through the strong interaction is expected to be an important discovery channel of SUSY, provided these particles are kinematically accessible at the LHC. The most stringent limits from the $7 \mathrm{TeV}$ 
and $8 \mathrm{TeV}$ runs of the LHC [2,3] exclude gluino masses up to $m_{\tilde{g}}=1.3 \mathrm{TeV}$ and superpartners of the quarks of the first two generations below $m_{\tilde{q}} \lesssim 875 \mathrm{GeV}$. Equal squark and gluino masses can be excluded up to $m_{\tilde{g}} \sim 1.7 \mathrm{TeV}$. First results at $\sqrt{s}=13 \mathrm{TeV}$ raised the mass bounds to $m_{\tilde{g}} \lesssim 1.75 \mathrm{TeV}$ and $m_{\tilde{q}} \lesssim 1.26 \mathrm{TeV}$ [4]. However, these bounds depend on assumptions, e.g. on the mass of the lightest supersymmetric particle and on decay chains, and can be evaded, for instance by compressed mass spectra or non-degenerate light-flavour squark masses. The search for SUSY therefore remains a focus of the 13$14 \mathrm{TeV}$ run of the LHC that has the potential to discover or exclude squarks and gluinos up to the $3 \mathrm{TeV}$ range. Turning exclusion limits on production cross sections into bounds on superparticle masses requires precise predictions for these cross sections, which motivates the computation of higher-order corrections to squark and gluino production. The next-to-leading order (NLO) corrections for production of the light-flavour squarks and gluinos in the supersymmetric extension of quantum chromodynamics (SQCD) have been known for a long time [5] and have been implemented in the program PROSPINO [6]. More recently, additional higher-order QCD corrections have been added to this result in various approximations [7-20]. Corresponding results for top squarks have been obtained as well [16, 21-28]. Complementary work to this improvement of total cross sections by higher-order QCD corrections is provided by the computation of electroweak contributions [29-36], the automation of NLO calculations in the MSSM [37, 38], the matching of NLO corrections to a parton shower [39-41], the calculation of NLO corrections to squark production and decay [40, 42, 43] and the estimate of finite-width effects [44].

The dominant production channels for squark and gluino production at hadron colliders are pair-production processes of the form

$$
N_{1} N_{2} \rightarrow \tilde{s} \tilde{s}^{\prime} X
$$

where $N_{1,2}$ denote the incoming hadrons and $\tilde{s}, \tilde{s}^{\prime}$ the two sparticles. In this paper we will consider all pair-production processes of gluinos and squarks except top squark production. The NLO SQCD corrections to squark and gluino production processes can become very large for heavy sparticle masses [5], up to $100 \%$ of the tree-level result for gluino-pair production. This raises the question of the convergence of the perturbative series. A substantial part of the large NLO corrections can be attributed to terms that are enhanced in the limit of a small relative velocity $\beta$ of the sparticles,

$$
\beta=\sqrt{1-\frac{\left(m_{\tilde{s}}+m_{\tilde{s}^{\prime}}\right)^{2}}{\hat{s}}} \rightarrow 0,
$$

where $\hat{s}$ is the partonic centre-of-mass energy. These corrections arise at each order in perturbation theory through threshold logarithms $\alpha_{s} \ln ^{2,1} \beta$ due to soft-gluon corrections and through Coulomb corrections of the form $\alpha_{s} / \beta$. The large NLO corrections to squark and gluino production and the significant contribution of the threshold region motivate the resummation of these threshold corrections, i.e. a reorganization of the perturbation theory under the assumption that both types of threshold corrections are of order one,

$$
\alpha_{s} \ln \beta \sim 1, \quad \frac{\alpha_{s}}{\beta} \sim 1 .
$$

The accuracy of the resummed perturbative series can be defined by representing the 
resummed cross section schematically as

$$
\begin{aligned}
\hat{\sigma}_{p p^{\prime}}= & \hat{\sigma}_{p p^{\prime}}^{(0)} \sum_{k=0}^{\infty}\left(\frac{\alpha_{s}}{\beta}\right)^{k}\left(1+\alpha_{s} c_{\mathrm{NNLL}}+\ldots\right) \\
& \times \exp [\underbrace{\ln \beta g_{0}\left(\alpha_{s} \ln \beta\right)}_{(\mathrm{LL})}+\underbrace{g_{1}\left(\alpha_{s} \ln \beta\right)}_{(\mathrm{NLL})}+\underbrace{\alpha_{s} g_{2}\left(\alpha_{s} \ln \beta\right)}_{(\mathrm{NNLL})}+\ldots] .
\end{aligned}
$$

Methods for the separate resummation of the two towers of corrections are well established and have been applied to squark and gluino production. The resummation of threshold logarithms [45-48] with a fixed-order treatment of Coulomb corrections was performed at NLL $[7,8,11,23]$ and more recently at NNLL accuracy $[15,18,20,26]$. The application of Coulomb-resummation [49] to squark and gluino production with a fixed-order treatment of threshold logarithms was considered in $[8,10,13,14]$.

In these approaches, only one of the two variables in (1.3) is considered to be of order one in the threshold region, which is not justified a priori. Therefore a combined resummation of soft and Coulomb corrections is desirable and was established in [12, 50] using effective-theory methods. The application of this method to squark and gluino production at NLL accuracy [16] has revealed a significant effect of Coulomb corrections and softCoulomb interference effects that can be as large as the soft corrections alone. Since the joint soft and Coulomb corrections at NLL can show an enhancement of up to $100 \%$ relative to the NLO cross section for some processes and large sparticle masses [16], a combined NNLL treatment seems to be required for a stabilisation of the perturbative behaviour. We note that when the Coulomb corrections are not summed, some of the sizeable corrections at NLL in the combined soft-Coulomb resummation appear only at the next order (NNLL) in pure soft-gluon resummation. Ref. [20] indeed confirms the earlier finding of a significant soft-Coulomb interference effect. In the present paper we perform for the first time such a combined soft and Coulomb resummation for squark and gluino production at NNLL accuracy. Preliminary results have been presented already in [19]. A combination of Coulomb corrections and NNLL soft resummation has also been performed for the case of top-squark bound states ("stoponium") in [27] using a formalism similar to ours.

With respect to our previous work on NNLL resummation for top quark production $[51,52]$, this paper contains several new theoretical results and features: we derive the extension of the spin-dependent non-Coulomb $\alpha_{s}^{2} \ln \beta$ terms given for top-pair production in [53] to squark and gluino production (these results have been quoted already in [19]). We also generalize the additional logarithm found in [54] for top-pair production to squark and gluino production and show how it arises in the effective-theory framework. For the soft-gluon resummation we use the scale choice introduced in [55] as a default. Our numerical cross section results are publicly available in the form of grids in the squark-gluino mass plane [1].

The paper is organized as follows: in section 2 we give an overview of squark and gluino production, review our resummation method and provide the input for NNLL resummation. We compute the single-logarithmic potential corrections and spell out our choice of the soft scale in soft-gluon resummation in the momentum-space framework. In section 3 we present our numerical results and specify our estimate of the remaining theoretical uncertainties. Some technical details of the NNLL resummation are provided in an appendix. 


\section{NNLL soft-Coulomb resummation for squark and gluino production}

\subsection{Production processes}

The total hadronic cross sections for the processes (1.1) can be obtained from short-distance production cross sections $\hat{\sigma}_{p p^{\prime}}\left(\hat{s}, \mu_{f}\right)$ for the partonic processes

$$
p p^{\prime} \rightarrow \tilde{s} \tilde{s}^{\prime} X, \quad p, p^{\prime} \in\{q, \bar{q}, g\},
$$

by a convolution with the parton luminosity functions $L_{p p^{\prime}}(\tau, \mu)$ :

$$
\sigma_{N_{1} N_{2} \rightarrow \tilde{s} \tilde{s}^{\prime} X}(s)=\int_{\tau_{0}}^{1} d \tau \sum_{p, p^{\prime}=q, \bar{q}, g} L_{p p^{\prime}}\left(\tau, \mu_{f}\right) \hat{\sigma}_{p p^{\prime}}\left(\tau s, \mu_{f}\right),
$$

with $\tau_{0}=4 M^{2} / s$ and the average sparticle mass

$$
M=\frac{m_{\tilde{s}}+m_{\tilde{s}^{\prime}}}{2} .
$$

The parton luminosity functions are defined in terms of the parton density functions (PDFs) as

$$
L_{p p^{\prime}}(\tau, \mu)=\int_{0}^{1} d x_{1} d x_{2} \delta\left(x_{1} x_{2}-\tau\right) f_{p / N_{1}}\left(x_{1}, \mu\right) f_{p^{\prime} / N_{2}}\left(x_{2}, \mu\right) .
$$

At leading order [56-58], the following partonic channels contribute to the production of light-flavour squarks and gluinos:

$$
\begin{array}{rlrl}
g g, q_{i} \bar{q}_{j} & \rightarrow \tilde{q} \overline{\tilde{q}}, & & \\
q_{i} q_{j} & \rightarrow \tilde{q} \tilde{q}, & \bar{q}_{i} \bar{q}_{j} \rightarrow \overline{\tilde{q}} \overline{\tilde{q}}, \\
g q_{i} & \rightarrow \tilde{g} \tilde{q}, & g \bar{q}_{i} & \rightarrow \tilde{g} \tilde{\tilde{q}}, \\
g g, q_{i} \bar{q}_{i} & \rightarrow \tilde{g} \tilde{g}, & &
\end{array}
$$

where $i, j=u, d, s, c, b$. Flavour indices of squarks have been suppressed. For the lightflavour squarks a common mass $m_{\tilde{q}}$ will be assumed. The predictions for the cross sections presented below always include a sum over the contributions of the ten light-flavour squarks $\left(\tilde{u}_{L / R}, \tilde{d}_{L / R}, \tilde{c}_{L / R}, \tilde{s}_{L / R}, \tilde{b}_{L / R}\right)$. The partonic cross sections for squark-anti-squark and squark-squark production differ for equal and unequal initial-state (anti-) quarks, but otherwise do not depend on the individual quark flavours. Therefore it is possible to express the cross section (2.2) in terms of diagonal and off-diagonal flavour-summed parton luminosities.

In this paper we consider higher-order corrections to partonic channels where the sparticle pair is dominantly produced with vanishing orbital momentum (i.e. in an $S$ wave), with a Born cross section $\hat{\sigma} \propto \beta$ in the threshold limit $\beta \rightarrow 0$. For the purpose of resummation, the partonic cross section $\hat{\sigma}_{p p^{\prime}}$ is decomposed into contributions of definite colour and spin of the final-state system. With regard to colour, the product of the $\mathrm{SU}(3)$ representations $r$ and $r^{\prime}$ of the initial state particles ( $R$ and $R^{\prime}$ of the final state particles) is decomposed into irreducible representations

$$
r \otimes r^{\prime}=\sum_{\alpha} r_{\alpha}, \quad R \otimes R^{\prime}=\sum_{R_{\alpha}} R_{\alpha} .
$$




\begin{tabular}{|c|c|c|c|c|}
\hline$\tilde{s} \tilde{s}^{\prime}$ & $p p^{\prime}$ & $\left(r_{\alpha}, R_{\beta}\right)$ & $S$ & Comments \\
\hline$\tilde{q} \overline{\tilde{q}}$ & $q \bar{q}$ & $(1,1),(8,8)$ & 0 & \\
& $g g$ & $(1,1),\left(8_{s}, 8\right)$ & 0 & \\
\hline$\tilde{q}_{i} \tilde{q}_{j}$ & $q q$ & $(\overline{3}, \overline{3})$ & 0 & $i \neq j$ only \\
& & $(6,6)$ & 0 & \\
\hline$\tilde{q} \tilde{g}$ & $q g$ & $(3,3),(\overline{6}, \overline{6}),(15,15)$ & $\frac{1}{2}$ & \\
\hline$\tilde{g} \tilde{g}$ & $q \bar{q}$ & $\left(8,8_{a}\right)$ & 1 & \\
& $g g$ & $(1,1),\left(8_{s}, 8_{s}\right),(27,27)$ & 0 & \\
\hline
\end{tabular}

Table 1. Spin and colour quantum numbers leading to $S$-wave production of squarks and gluinos.

For squark and gluino production the relevant decompositions are

$$
\begin{aligned}
& 3 \otimes \overline{3}=1 \oplus 8, \\
& 3 \otimes 3=\overline{3} \oplus 6, \\
& 3 \otimes 8=3 \oplus \overline{6} \oplus 15, \\
& 8 \otimes 8=1 \oplus 8_{s} \oplus 8_{a} \oplus 10 \oplus \overline{10} \oplus 27 .
\end{aligned}
$$

The production cross sections can be decomposed into a colour basis characterized by pairs of representations, $P_{i}=\left(r_{\alpha}, R_{\beta}\right)$ with equivalent initial- and final-state representations, $r_{\alpha} \sim R_{\beta}$. Basis tensors for the pairs $P_{i}$ can be constructed in terms of Clebsch-Gordan coefficients [50]. The colour and spin quantum numbers resulting in $S$-wave sparticle production have been classified e.g. in [59], see also [13] for gluino pair production. The results are collected in table 1.

The higher-order corrections are written in terms of scaling functions $f_{p p^{\prime}}^{(n)}$ as

$$
\hat{\sigma}_{p p^{\prime}}=\sum_{i} \sum_{S=\left|s-s^{\prime}\right|}^{s+s^{\prime}} \hat{\sigma}_{p p^{\prime}, i}^{(0), S}\left[1+\sum_{n=1}^{\infty}\left(\frac{\alpha_{s}}{4 \pi}\right)^{n} f_{p p^{\prime}, i}^{(n), S}\right] .
$$

Here the sum over $i$ runs over the colour basis defined by the pairs $P_{i}$, while $s\left(s^{\prime}\right)$ is the spin of the sparticle $\tilde{s}\left(\tilde{s}^{\prime}\right)$ and $S$ the total spin of the sparticle pair.

The colour-separated Born cross sections $\hat{\sigma}_{p p^{\prime}, i}^{(0)}$ for squark and gluino production are available in $[8,11,23]$. The colour-averaged NLO scaling functions were computed in [5] for degenerate light-flavour squark masses and have been implemented in the computer program PROSPINO [6]. For general squark spectra, the NLO corrections have been computed recently $[37,39]$. An approximation of the NNLO scaling functions consisting of all terms that are enhanced in the limit $\beta \rightarrow 0$ has been given in [53], up to an additional $\alpha_{s}^{2} \ln \beta$ term that has been calculated for the case of top-quark production in [54]. In section 2.3 we derive the generalization of this contribution for the production of squarks and gluinos. 


\subsection{Resummation formula}

Up to NNLL accuracy, the partonic production cross sections for the processes (2.5) factorize in the threshold limit $\beta \rightarrow 0$ into spin- and colour-dependent hard and Coulomb functions $H_{i}^{S}$ and $J_{R_{\alpha}}^{S}$ and a soft function $W^{R_{\alpha}}$ depending only on the total colour charge $R_{\alpha}$ of the final-state particles [12, 50]:

$$
\hat{\sigma}_{p p^{\prime}}\left(\hat{s}, \mu_{f}\right)=\sum_{i} \sum_{S=\left|s-s^{\prime}\right|}^{s+s^{\prime}} H_{i}^{S}\left(m_{\tilde{q}}, m_{\tilde{g}}, \mu_{f}\right) \int d \omega J_{R_{\alpha}}^{S}\left(E-\frac{\omega}{2}\right) W_{i}^{R_{\alpha}}\left(\omega, \mu_{f}\right) .
$$

Here $E=\sqrt{\hat{s}}-2 M$ is the partonic centre-of-mass energy measured from threshold. The hard function encodes the partonic hard-scattering processes and is related to squared on-shell scattering amplitudes at threshold. The potential function is defined in terms of non-relativistic fields for the sparticles whose interactions are described in potential nonrelativistic QCD (PNRQCD). Solving the Schrödinger equation in PNRQCD allows to sum the Coulomb corrections to all orders. The soft function is defined in terms of soft Wilson lines and contains the threshold logarithms. The convolution of the soft- and potential functions accounts for the energy loss of the squark/gluino system due to soft gluons with energy of the order $M \beta^{2}$. For the colour basis based on the pairs of representations $P_{i}$ constructed in [50], the soft function is diagonal in colour space and identical to that of a simpler two-to-one scattering process where a single heavy particle with colour charge $R_{\alpha}$ is produced from the two incoming partons. This basis has been assumed in writing (2.9). Only production channels with an $S$-wave contribution will be taken into account in (2.9). It can be seen from table 1 that only a single spin quantum number contributes for the threshold production for a given partonic colour channel. In practice the spin sum in (2.8) therefore collapses to a single term, so the sum over $S$ and the spin label on the hard function will be suppressed in the following.

Resummation of threshold logarithms is performed by evolving the soft function from a soft scale $\mu_{s} \sim M \beta^{2}$ to a hard-scattering scale $\mu_{f} \sim M$ using a renormalization-group equation. The anomalous dimensions required for NNLL resummation are collected in [50]. The hard function is evolved from a scale $\mu_{h} \sim 2 M$ to $\mu_{f}$. In the momentum-space formalism [60, 61] the resummed cross section can be written as [12]

$$
\begin{aligned}
\hat{\sigma}_{p p^{\prime}}^{\mathrm{res}}\left(\hat{s}, \mu_{f}\right)= & \sum_{i} H_{i}\left(m_{\tilde{q}}, m_{\tilde{g}}, \mu_{h}\right) U_{R_{\alpha}}\left(\mu_{h}, \mu_{s}, \mu_{f}\right)\left(\frac{2 M}{\mu_{s}}\right)^{-2 \eta} \\
& \times \tilde{s}_{i}^{R_{\alpha}}\left(\partial_{\eta}, \mu_{s}\right) \frac{e^{-2 \gamma_{E} \eta}}{\Gamma(2 \eta)} \int_{0}^{\infty} d \omega \frac{J_{R_{\alpha}}^{S}\left(M \beta^{2}-\frac{\omega}{2}\right)}{\omega}\left(\frac{\omega}{\mu_{s}}\right)^{2 \eta} .
\end{aligned}
$$

Here the energy variable in the argument of the potential function has been expanded near threshold which yields the non-relativistic expression $E=M \beta^{2}$. This defines our default implementation. The derivation of the NLO potential function required at NNLL accuracy is the subject of section 2.3 and the result is given in (2.46) below. The quantity $\tilde{s}_{i}^{R_{\alpha}}$ is the Laplace transform of the soft function. For NNLL resummation, the NLO soft function [50] 
is required which reads

$$
\begin{aligned}
\tilde{s}_{i}^{R_{\alpha}}(\rho, \mu) & =\int_{0}^{\infty} d \omega e^{-s \omega} W_{i}^{R_{\alpha}}(\omega, \mu) \\
& =1+\frac{\alpha_{s}}{4 \pi}\left[\left(C_{r}+C_{r^{\prime}}\right)\left(\rho^{2}+\frac{\pi^{2}}{6}\right)-2 C_{R_{\alpha}}(\rho-2)\right]+\mathcal{O}\left(\alpha_{s}^{2}\right),
\end{aligned}
$$

with $s=1 /\left(e^{\gamma_{E}} \mu e^{\rho / 2}\right)$. After carrying out the differentiations with respect to $\eta$ in $(2.10)$, this variable is identified with a resummation function which contains single logarithms, $\eta=\frac{2 \alpha_{s}}{\pi}\left(C_{r}+C_{r^{\prime}}\right) \ln \left(\mu_{s} / \mu_{f}\right)+\ldots$, while the resummation function $U_{i}$ sums the Sudakov double logarithms $\alpha_{s} \ln ^{2} \frac{\mu_{h}}{\mu_{f}}$ and $\alpha_{s} \ln ^{2} \frac{\mu_{s}}{\mu_{f}}$. The precise definitions of these functions for the case of heavy-particle pair production are given in [12] and the expansions required for NNLL accuracy can be found in [61]. For $\mu_{s}<\mu_{f}$ the function $\eta$ is negative and the factor $\omega^{2 \eta-1}$ in the resummed cross section (2.10) has to be understood in the distributional sense, as discussed in detail in [51]. The prescription for the choice of the soft scale is detailed in section 2.4.

\subsubsection{Hard functions}

The perturbative expansion of the hard function in the resummation formula (2.10) in the $\overline{\mathrm{MS}}$ scheme can be written as

$$
H_{i}\left(m_{\tilde{q}}, m_{\tilde{g}}, \mu\right)=H_{i}^{(0)}\left(m_{\tilde{q}}, m_{\tilde{g}}, \mu\right)\left[1+\sum_{n}\left(\frac{\alpha_{s}(\mu)}{4 \pi}\right)^{n} h_{i}^{(n)}\left(m_{\tilde{q}}, m_{\tilde{g}}, \mu\right)\right],
$$

where for NNLL resummation the one-loop coefficients $h_{i}^{(1)}$ are required.

The leading-order hard function $H_{i}^{(0)}$ is related to the threshold limit of the Born cross section for a given colour channel according to [12]

$$
\hat{\sigma}_{p p^{\prime}}^{(0) R_{\alpha}}(\hat{s}) \underset{\hat{s} \rightarrow 4 M^{2}}{=} \frac{\left(m_{\tilde{s}} m_{\tilde{s}^{\prime}}\right)^{3 / 2}}{M} \frac{\beta}{2 \pi} H_{i}^{(0)}+\mathcal{O}\left(\beta^{3}\right) .
$$

In our numerical implementation, we define the leading-order hard functions $H_{i}^{(0)}$ in terms of the exact Born-cross sections, instead of the leading term in the threshold limit, which is seen to improve the accuracy of the threshold approximation in some cases, but not in a systematic fashion. However, the hard function for a given production and colour channel is set to zero if there is no $S$-wave contribution to the Born cross section at threshold, even if the full Born cross section for this channel is non-vanishing. This affects the sub-process $q \bar{q} \rightarrow \tilde{g} \tilde{g}$ in the singlet and symmetric octet channels, the sub-processes $g g \rightarrow \tilde{g} \tilde{g}$ and $g g \rightarrow \tilde{q} \overline{\tilde{q}}$ in the anti-symmetric octet channel, as well as $q_{i} q_{i} \rightarrow \tilde{q}_{i} \tilde{q}_{i}$ in the triplet channel, see table 1 .

A prescription to compute the one-loop hard functions from on-shell Born and oneloop amplitudes at threshold has been given in [12]. Alternatively, the one-loop coefficient can be read off from the constant term in the threshold expansion of the total NLO cross section given in (A.1). This allows to extract the one-loop hard functions from recent computations of the corresponding matching coefficients in the Mellin-space approach to 
threshold resummation [15, 59], which are defined as the constant term in the Mellintransformed one-loop cross section in the threshold limit (for gluino-pair production, see also $[13,17])$. From the Mellin transformation of the NLO threshold cross section in momentum space (A.1), we obtain the relation of the one-loop hard coefficients $h_{i}^{(1)}$ to the matching coefficients $\mathcal{C}_{p p^{\prime} \rightarrow \tilde{s} \tilde{s}^{\prime}, I}^{(1)}$ in the notation of $[15,59]$

$$
\begin{aligned}
h_{i}^{(1)}\left(m_{\tilde{q}}, m_{\tilde{g}}, \mu\right)= & -4\left(C_{r}+C_{r^{\prime}}\right)\left(\ln ^{2}\left(\frac{2 M}{\mu e^{\gamma_{E}}}\right)+\frac{\pi^{2}}{24}\right) \\
& +4 C_{R_{\alpha}}\left(\ln \left(\frac{2 M}{\mu e^{\gamma_{E}}}\right)-1\right)+4 \mathcal{C}_{p p^{\prime} \rightarrow \tilde{s} \tilde{s}^{\prime}, I}^{(1)}\left(m_{\tilde{q}}, m_{\tilde{g}}, \mu\right),
\end{aligned}
$$

where $I$ is the label of the colour basis tensors used in [59] that correspond to the basis elements $P_{i}$ in our notation.

In addition to the dependence on the scale $\mu$ and the squark and gluino masses as indicated in (2.14), the one-loop hard functions in SQCD depend as well on the top-quark mass, with all other quarks treated as massless. Numerical results for the coefficients $\mathcal{C}_{p p^{\prime} \rightarrow \tilde{s} \tilde{s}^{\prime}, I}^{(1)}$ have been plotted in $[15,59]$. In the case of gluino-pair production and squarkgluino production, the hard functions become singular for $m_{\tilde{g}}=m_{\tilde{q}}+m_{t}$ when the on-shell decay-channel $\tilde{g} \rightarrow \tilde{t} t$ opens up. ${ }^{1}$ This singularity is not physical and arises from neglecting the gluino decay width. In addition, for gluino-pair production from a quark-antiquark initial state, the threshold limit of the Born hard function goes to zero for $m_{\tilde{q}}=m_{\tilde{g}}$, so that the $S$-wave contribution to this channel vanishes in this special point of parameter space. As a result, the relative NLO corrections given by the one-loop hard coefficient diverge. Since we only apply resummation to the $S$-wave production channel, we set the resummed contribution of the quark-antiquark initial state to zero for $m_{\tilde{q}}=m_{\tilde{g}}$, while it is included in fixed-order at NLO through the matching to PROSPINO. In practice, this prescription is implemented by using the threshold limit of the Born hard function $H^{(0)}$ for the subprocess $q \bar{q} \rightarrow \tilde{g} \tilde{g}$ for $0.9<m_{\tilde{q}} / m_{\tilde{g}}<1.1$. The numerical effect of the precise choice of this interval is negligible.

\subsection{Potential effects}

In the framework of $[12,50]$, the non-relativistic sparticles are described by the Lagrangian of potential non-relativistic SQCD (PNRSQCD). To the order relevant for NNLL resummation, the Lagrangian reads ${ }^{2}$

$$
\begin{aligned}
\mathcal{L}_{\mathrm{PNRSQCD}}= & \psi^{\dagger}\left(i D_{s}^{0}+\frac{\vec{\partial}^{2}}{2 m_{\tilde{s}}}+\frac{\vec{\partial}^{4}}{8 m_{\tilde{s}}^{3}}\right) \psi+\psi^{\dagger}\left(i D_{s}^{0}+\frac{\vec{\partial}^{2}}{2 m_{\tilde{s}^{\prime}}}+\frac{\vec{\partial}^{4}}{8 m_{\tilde{s}^{\prime}}^{3}}\right) \psi^{\prime} \\
& -\frac{1}{2^{\delta_{\tilde{s} \tilde{s}^{\prime}}}} \int d^{3} \vec{r} V_{\{k\}}(\vec{r}, \vec{\partial}) \psi_{k_{4}}^{\prime \dagger}(x) \psi_{k_{3}}^{\dagger}(x+\vec{r}) \psi_{k_{1}}(x+\vec{r}) \psi_{k_{2}}^{\prime}(x) .
\end{aligned}
$$

\footnotetext{
${ }^{1}$ Note that in the NLO calculations of $[5,59]$ virtual top squarks are treated as mass-degenerate with the light-flavour squarks.

${ }^{2}$ Note that the sign of the potential term in [12] is incorrect, which, however, has no consequence for the results presented there. For the case of fermions, the sign here is consistent with [62] if the different conventions for antiparticles are taken into account.
} 
Here the fields $\psi_{k}^{\dagger}$ and $\psi_{k}^{\prime \dagger}$ are non-relativistic fields which create the heavy sparticles $\tilde{s}$ and $\tilde{s}^{\prime}$. The label $k$ collectively denotes the flavour, spin and colour quantum numbers of the non-relativistic field, $\psi_{k}=\psi_{n, a, \alpha}$, where Latin letters $n$ and $a$ are used for flavour and colour indices, respectively, while the Greek index $\alpha$ denotes the spin index of the field. For objects such as the potential, which depend on the labels of several fields, we employ a multi-index convention for the spin indices, $\{\alpha\}=\alpha_{1} \alpha_{2} \alpha_{3} \alpha_{4}$, and analogously for the colour $(\{a\})$, flavour $(\{n\})$, and collective $(\{k\})$ index. The soft gluon field couples to the nonrelativistic sparticles through the soft covariant derivative $i D_{s}^{0} \psi=\left(i \partial^{0}+g_{s} \mathbf{T}^{(R) a} A^{a 0}\right) \psi$, where $\mathbf{T}^{(R) a}$ are the $\mathrm{SU}(3)$ generators in the representation $R$. Note that a factor $1 / 2$ appears in the potential in the case of identical sparticle species, where we treat particles as identical that belong to the same spin and $\mathrm{SU}(3)$ representation, and species (that is, the ten light-flavour squarks are treated as identical particles with an index $n$ denoting flavour and the helicity label).

For NNLL accuracy, higher-order potential effects beyond the leading Coulomb potential have to be taken into account, see [62] for a detailed discussion in the PNRQCD formalism used here. The relevant potentials are given by the NLO Coulomb potential, the $1 / m^{2}$ corrections to the tree-level potential, the one-loop $1 / m$ potential, and the so-called annihilation contributions,

$$
\begin{aligned}
V_{\{k\}}= & \mathbf{T}_{a_{3} a_{1}}^{(R) a} \mathbf{T}_{a_{4} a_{2} a}^{\left(R^{\prime}\right)}\left[V_{\mathrm{C}} \delta_{\alpha_{3} \alpha_{1}} \delta_{\alpha_{4} \alpha_{2}}+\delta_{1 / m^{2}} V_{\{\alpha\}}\right] \delta_{n_{3} n_{1}} \delta_{n_{4} n_{2}} \\
& +\delta_{1 / m} V_{\{a\}} \delta_{\alpha_{3} \alpha_{1}} \delta_{\alpha_{4} \alpha_{2}} \delta_{n_{3} n_{1}} \delta_{n_{4} n_{2}}+\delta_{\mathrm{ann}} V_{\{k\}} .
\end{aligned}
$$

Note that due to $(1.3), \mathcal{O}(\beta)$ and $\mathcal{O}\left(\alpha_{s} \beta, \beta^{2}\right)$ suppressed potentials appear here on the same footing, if the latter generate a logarithm of $\beta$. All contributions to the potential apart from the annihilation contribution are flavour-independent, while only the $1 / \mathrm{m}^{2}$ potential and the annihilation contribution are spin-dependent. Following [12, 50], we perform a projection of the potential on states with definite colour charge and spin of the heavy particle system by introducing projectors $P_{\{a\}}^{R_{\alpha}}$ and $\Pi_{\{\alpha\}}^{S}$ on colour and spin space, respectively. The colour projectors can be written in terms of Clebsch-Gordan coefficients for the combination of the representations $R$ and $R^{\prime}$ into the irreducible representation $R_{\alpha}$,

$$
P_{a_{1} a_{2} a_{3} a_{4}}^{R_{\alpha}}=C_{A a_{1} a_{2}}^{R_{\alpha *}} C_{A a_{3} a_{4}}^{R_{\alpha}},
$$

where the index $A$ is the colour index for the irreducible representation $R_{\alpha}$.

Following the reasoning of appendix A of [50], gauge invariance implies that the potential can be expanded in terms of the colour projectors $(2.17)^{3}$

$$
V_{\{k\}}=\sum_{R_{\alpha}} V_{\{n, \alpha\}}^{R_{\alpha}} P_{a_{3} a_{4} a_{1} a_{2}}^{R_{\alpha}}
$$

\footnotetext{
${ }^{3}$ Strictly speaking these arguments imply that the potential can be written in the form $V_{\{a\}}=$ $\sum_{I} V^{I} C_{A a_{3} a_{4}}^{R_{\alpha *}} C_{A a_{1} a_{2}}^{R_{\beta}}$ where the sum is over pairs $P_{I}=\left(R_{\alpha}, R_{\beta}\right)$ of equivalent representations $R_{\alpha} \sim R_{\beta}$. In squark-gluino production, the only case where equivalent but non-identical representations appear is the production of gluino pairs, that can be in an $8_{s}$ or $8_{a}$ state. However, for a given partonic initial state, only one of the two channels appears (see table 1), so in practice it is sufficient to consider the case where the two representations are identical and the decomposition assumes the form (2.18).
} 
We will also only require potentials which allow for an analogous decomposition in spin space. The potential term in the Lagrangian then assumes the form

$$
V_{\{k\}} \psi_{k_{4}}^{\prime \dagger} \psi_{k_{3}}^{\dagger} \psi_{k_{1}} \psi_{k_{2}}^{\prime}=\sum_{R_{\alpha}, S} V_{\{n\}}^{R_{\alpha}, S}\left[\left(\psi \otimes \psi^{\prime}\right)_{n_{3} n_{4}}^{R_{\alpha}, S}\right]^{\dagger}\left(\psi \otimes \psi^{\prime}\right)_{n_{1} n_{2}}^{R_{\alpha}, S}
$$

in tensor-product notation $\left(\psi \otimes \psi^{\prime}\right)_{12}(t, \vec{r}, \vec{R})=\psi_{1}^{(0)}\left(\vec{R}+\frac{\vec{r}}{2}\right) \psi_{2}^{\prime(0)}\left(\vec{R}-\frac{\vec{r}}{2}\right)$.

As shown in [12] for the case of the Coulomb potential, the interaction of the nonrelativistic particles with soft gluons can be eliminated from the PNR(S)QCD Lagrangian through a field redefinition. It can be seen that the same transformation also decouples soft gluons from a general gauge invariant potential. Therefore the fields in the Lagrangian $(2.15)$ can be replaced by the decoupled fields $\psi^{(0)}$ and the covariant derivatives can be replaced by ordinary derivatives. This decoupling holds to all orders in the strong coupling but at leading power in the non-relativistic expansion in $\beta$. Non-decoupling effects appear at $\mathcal{O}(\beta)$ through the chromo-electric interaction, but do not contribute NNLL corrections to the total cross section [12].

The potential function is defined as the correlation function of the decoupled potential fields,

$$
J_{\{k\}}(q)=\sum_{R_{\alpha}, S} P_{a_{3} a_{4} a_{1} a_{2}}^{R_{\alpha}} \Pi_{\alpha_{3} \alpha_{4} \alpha_{1} \alpha_{2}}^{S} J_{R_{\alpha},\{n\}}^{S}(q) .
$$

For identical bosonic (fermionic) sparticles, the potential function satisfies the symmetry (antisymmetry) property

$$
J_{1234}= \pm J_{2134}= \pm J_{1243}
$$

that implies the symmetry properties of the colour and spin-projected potential function $J_{R_{\alpha},\{n\}}^{S}$ together with the symmetry or antisymmetry of the colour and spin representations. Since the Coulomb potential, the one-loop $1 / m$ and the tree-level $1 / m^{2}$ potentials are flavour-independent, the flavour structure can be neglected in all contributions apart from the annihilation contribution, which will be discussed in section 2.3.2.

The LO potential function, which resums all corrections of the form $\left(\alpha_{s} / \beta\right)^{n}$, is given by the imaginary part of the zero-distance Green function of the Schrödinger equation with the leading Coulomb potential, i.e. the $\mathcal{O}\left(\alpha_{s} / \beta\right)$ contribution to $(2.24)$,

$$
J_{R_{\alpha}}^{S,(0)}(E)=2 \operatorname{Im}\left[G_{R_{\alpha}}^{(0)}(0,0 ; E)\right] .
$$

The explicit expression can be obtained by the simple replacement $m_{t} \rightarrow 2 m_{\text {red }}$ from the corresponding result for top-pair production quoted e.g. in eq. (A.1) of [51]. For the NNLL prediction, the NLO contributions to the potential (2.16) are taken into account perturbatively,

$$
\delta G_{R_{\alpha},\{n\}}^{(1), S}(0,0, E)=\int d^{3} z G_{R_{\alpha}}^{(0)}(0, \vec{z}, E)\left(i \delta V_{\{n\}}^{R_{\alpha}, S}(\vec{z})\right) i G_{R_{\alpha}}^{(0)}(\vec{z}, 0, E),
$$

where it was used that all NLO potentials are diagonal with respect to the colour representations and that the leading Coulomb Green function is spin-independent. It is not necessary to (anti-)symmetrize the Green function with respect to the flavour indices. The 
contribution to the cross section automatically inherits the correct symmetry properties from those of the potential and the hard function. This allows us to omit the flavour indices of $G_{R_{\alpha}}^{(0)}$. The solution to $(2.23)$ for the potential (2.16) is given in section 2.3.3.

\subsubsection{Coulomb and non-Coulomb potential terms}

In momentum space, the colour-projected Coulomb potential up to NLO reads

$$
\tilde{V}_{\mathrm{C}}^{R_{\alpha}}(\boldsymbol{p}, \boldsymbol{q})=\frac{4 \pi D_{R_{\alpha}} \alpha_{s}(\mu)}{\boldsymbol{q}^{2}}\left[1+\frac{\alpha_{s}(\mu)}{4 \pi}\left(a_{1}-\beta_{0} \ln \frac{\boldsymbol{q}^{2}}{\mu^{2}}\right)+\ldots\right],
$$

where $\beta_{0}=\frac{11}{3} C_{A}-\frac{4}{3} n_{l} T_{f}$ is the one-loop beta-function coefficient, and $a_{1}=\frac{31}{9} C_{A}-\frac{20}{9} n_{l} T_{f}$. The coefficient $D_{R_{\alpha}}$ of the Coulomb potential for a pair of heavy particles in the SU(3) representations $R, R^{\prime}$ in the irreducible product representation $R_{\alpha}$ is given in terms of the quadratic Casimir operators for the various representations by

$$
D_{R_{\alpha}}=\frac{1}{2}\left(C_{R_{\alpha}}-C_{R}-C_{R^{\prime}}\right)
$$

where negative values correspond to an attractive Coulomb potential, positive values to a repulsive one. The numerical values for the representations relevant for squark and gluino production can be found in $[12,63]$.

The following potentials are all suppressed by two powers of velocity in the nonrelativistic expansion, but have to be considered, since, contrary to the Coulomb potential, they generate logarithms of $\beta$ not related to the running coupling. At the next order in $1 / m$, the colour-projected one-loop potential of order $m^{-1}$ in $D=4-2 \epsilon$ dimensions is given by

$$
\begin{aligned}
\delta_{1 / m} \tilde{V}^{R_{\alpha}}(\mathbf{q})= & \frac{\pi^{2} \alpha_{s}^{2} D_{R_{\alpha}}}{2 m_{\mathrm{red}}} \frac{\mu^{2 \epsilon}}{|\mathbf{q}|^{1+2 \epsilon}} \frac{e^{\epsilon \gamma_{E}} \Gamma^{2}\left(\frac{1}{2}-\epsilon\right) \Gamma\left(\frac{1}{2}+\epsilon\right)}{\pi^{3 / 2} \Gamma(1-2 \epsilon)} \\
& \times\left(\frac{D_{R_{\alpha}}}{2}(1-2 \epsilon) \frac{2 m_{\mathrm{red}}}{M}+C_{A}(1-\epsilon)\right) .
\end{aligned}
$$

We obtain the $1 / \mathrm{m}^{2}$ potential at tree-level for squark-and gluino production from the generalization of the spin-dependent non-Coulomb terms for top-quark production [51, 53] to squarks and gluinos (the result has been quoted already in [19]). This derivation is analogous to the one for the $1 / \mathrm{m}^{2}$ potential for threshold production of top-quark pairs. Details for the latter can be found in [62]. The general expression is

$$
\begin{aligned}
\delta_{1 / m^{2}} \tilde{V}^{R_{\alpha}}(\boldsymbol{p}, \boldsymbol{q})= & \frac{4 \pi D_{R} \alpha_{s}\left(\mu^{2}\right)}{\boldsymbol{q}^{2}}\left[\frac{\mathbf{p}^{2}}{m_{\tilde{s}} m_{\tilde{s}^{\prime}}}-\frac{\mathbf{q}^{2}}{8 m_{\tilde{s}}^{2} m_{\tilde{s}^{\prime}}^{2}}\left(2 m_{\tilde{s}} m_{\tilde{s}^{\prime}}+m_{\tilde{s}}^{2} c_{2}^{\tilde{s}^{\prime}}+m_{\tilde{s}^{\prime}}^{2} c_{2}^{\tilde{s}}\right)\right. \\
& +\frac{c_{2}^{\tilde{s}} c_{2}^{\tilde{s}^{\prime}}}{16 m_{\tilde{s}} m_{\tilde{s}^{\prime}}}\left[\sigma^{i}, \sigma^{j}\right] q^{j} \otimes\left[\sigma^{i}, \sigma^{k}\right] q^{k}+c_{2}^{\tilde{s}}\left(\frac{1}{8 m_{\tilde{s}}^{2}}+\frac{1}{4 m_{\tilde{s}} m_{\tilde{s}^{\prime}}}\right)\left[\sigma^{i}, \sigma^{j}\right] q^{i} p^{j} \otimes \mathbf{1} \\
& \left.+c_{2}^{\tilde{s}^{\prime}}\left(\frac{1}{8 m_{\tilde{s}^{\prime}}^{2}}+\frac{1}{4 m_{\tilde{s}} m_{\tilde{s}^{\prime}}}\right) \mathbf{1} \otimes\left[\sigma^{i}, \sigma^{j}\right] q^{i} p^{j}\right],
\end{aligned}
$$

with 1 the $2 \times 2$ unit matrix in spin space, $\boldsymbol{q}=\boldsymbol{p}^{\prime}-\boldsymbol{p}$, and $\boldsymbol{p}\left(\boldsymbol{p}^{\prime}\right)$ the in-going (out-going) three-momentum of the heavy particle in the scattering amplitude. The coefficient $c_{2}$ has 
the tree-level value zero (one) for scalar (fermionic) sparticles. For scalars it also sets the corresponding spin-dependent terms to zero.

Projecting on the relevant spin states (see section 4.5 of [62]) and setting $D \rightarrow 4$, which is justified when one is only interested in the logarithmically enhanced term generated by the potential insertion, the non-Coulomb corrections can be cast in the form

$$
\delta_{1 / m^{2}} \tilde{V}^{R_{\alpha}, S}(\boldsymbol{p}, \boldsymbol{q})=\frac{4 \pi D_{R_{\alpha}} \alpha_{s}}{\mathbf{q}^{2}}\left[\frac{\mathbf{p}^{2}}{m_{\tilde{s}} m_{\tilde{s}^{\prime}}}+\frac{\mathbf{q}^{2}}{4 m_{\mathrm{red}}^{2}} \nu_{\text {spin }}^{S}\right],
$$

where the spin-dependent coefficient for the squark and gluino production processes is given by

$$
\begin{aligned}
\nu_{\text {spin }}(\tilde{q} \tilde{\tilde{q}})= & \nu_{\text {spin }}(\tilde{q} \tilde{q})=-\frac{2 m_{\text {red }}}{4 M}, & & \nu_{\text {spin }}^{s=\frac{1}{2}}(\tilde{q} \tilde{g})=\frac{1}{2}\left(\frac{m_{\tilde{g}}^{2}}{\left(m_{\tilde{q}}+m_{\tilde{g}}\right)^{2}}-1\right), \\
\nu_{\text {spin }}^{S=0}(\tilde{g} \tilde{g}) & =0, & \nu_{\text {spin }}^{S=1}(\tilde{g} \tilde{g}) & =-\frac{2}{3} .
\end{aligned}
$$

Together with the Coulomb potential (2.24) and the $1 / m$ potential in (2.26), this result is the needed generalization of eq. (4.95) of [62], up to the so-called annihilation contribution, which is derived in the next section.

Using these results we can now determine the corresponding logarithm of $\beta$ in the NNLO cross section. For this purpose we use the known results for the NNLO Green function of a system of two particles with equal masses from [64] (given explicitly in [65]) and generalize them to the case of unequal masses. In the following, we briefly outline this derivation, leaving a more detailed description for a momentum independent potential to section 2.3.2. It is straightforward to adapt the coefficients of the potentials in the expressions for the Green function to the more general case. Afterwards, the remaining mass dependence is due to the equation of motion and thus has to be identified with the reduced mass. We then expand the expressions to order $\alpha_{s}^{2}$, keeping only logarithms of $\beta$. Note that in addition to the $1 / \mathrm{m}$ and $1 / \mathrm{m}^{2}$ potentials discussed above, we also have to include the kinetic energy correction $\mathbf{p}^{4} /\left(8 m_{\tilde{s}}^{3}\right)$ from the terms with a fourth power of the spatial derivative in (2.15). The final result reads

$$
\begin{aligned}
\Delta \hat{\sigma}_{p p^{\prime}, \mathrm{nC}}^{(2) R_{\alpha}, S}\left(\hat{s}, \mu_{f}\right) & =\hat{\sigma}_{p p^{\prime}}^{(0) R_{\alpha}, S}(\hat{s}) \alpha_{s}^{2} \ln \beta\left[-D_{R_{\alpha}} b_{1}-2 D_{R_{\alpha}}^{2}\left(1+\nu_{\mathrm{spin}}^{S}+\frac{m_{\mathrm{red}}}{2 M}\right)\right] \\
& =\hat{\sigma}_{p p^{\prime}}^{(0) R_{\alpha}, S}(\hat{s}) \alpha_{s}^{2} \ln \beta\left[C_{A} D_{R_{\alpha}}-2 D_{R_{\alpha}}^{2}\left(1+\nu_{\mathrm{spin}}^{S}\right)\right]
\end{aligned}
$$

where $b_{1}=-C_{A}-D_{R_{\alpha}} m_{\text {red }} / M$ is the 1-loop coefficient of the $1 / m$ potential, cf. (2.26). Combining the contributions of the $1 / \mathrm{m}$ and $1 / \mathrm{m}^{2}$ potential, we obtain the same expression as in eq. (3) of [53], which was derived for the equal mass case. Remarkably, even for the case of unequal masses, the process dependence is completely contained in the coefficient $\nu_{\text {spin }}^{S}$ and the leading order cross section.

\subsubsection{Annihilation contributions}

We next derive the annihilation contribution $\delta_{\text {ann }} \tilde{V}_{\{k\}}$ to the potential (2.16) for the squark and gluino pair-production processes as well as the resulting corrections to the potential 


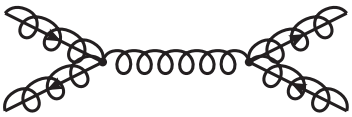

(a)

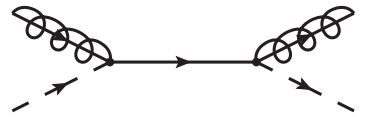

(b)

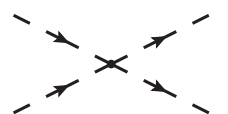

(c)

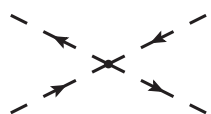

$(d)$

Figure 1. Tree-level contributions to the matching of four-field annihilation operators in NR(S)QCD. Dashed lines represent scalars, solid lines quarks and the solid-curly lines the gluino.

function (2.46) and the threshold expansion of the NNLO cross section. For the case of top-quark pair production this single-logarithmic correction of order $\alpha_{s}^{2} \ln \beta$ appears only in the $q \bar{q}$ partonic channel. It has been identified in [54] and was not included in the approximate NNLO cross section of [53].

The annihilation corrections arise from four-field operators in NRSQCD that match onto a local contribution to the potential (2.16) in PNRSQCD and contribute to the cross section at NNLO provided their matching coefficients are generated at tree level. The matching coefficients are obtained by equating the EFT matrix element with an insertion of the potential to the non-relativistic expansion of the matrix element of the two-to-two sparticle scattering process $\tilde{s} \tilde{s}^{\prime} \rightarrow \tilde{s} \tilde{s}^{\prime}$,

$$
\begin{aligned}
\frac{1}{2^{\delta_{\tilde{s} \tilde{s}^{\prime}}}}(-i) \delta_{\mathrm{ann}} \tilde{V}_{\{k\}}\left\langle\tilde{s}_{3} \tilde{s}_{4}^{\prime}\left|\psi_{k_{4}}^{\prime \dagger} \psi_{k_{3}}^{\dagger} \psi_{k_{1}} \psi_{k_{2}}^{\prime}\right| \tilde{s}_{1} \tilde{s}_{2}^{\prime}\right\rangle_{\mathrm{EFT}} \\
\quad=\left.\frac{1}{4 \sqrt{m_{\tilde{s}_{1}} m_{\tilde{s}_{2}^{\prime}} m_{\tilde{s}_{3}} m_{\tilde{s}_{4}^{\prime}}}} i \mathcal{M}\left(\tilde{s}_{1} \tilde{s}_{2}^{\prime} \rightarrow \tilde{s}_{3} \tilde{s}_{4}^{\prime}\right)\right|_{\hat{s}=4 M^{2}} ^{\mathrm{ann}}
\end{aligned}
$$

where the pre-factor on the right-hand side arises from the non-relativistic normalization of the one-particle states. As indicated by the superscript "ann", only the contributions to the matrix element matching to a local four-fermion operator must be taken into account. Also $t$-channel gluon exchange contributions are excluded since they are assigned to the non-Coulomb scattering potential. The relevant tree-level diagrams for the various squark and gluino pair-production processes are shown in figure 1. Diagram $(a)$ is the typical diagram for fermion-antifermion annihilation through a gluon, which arises for the gluinogluino process. Diagram $(b)$ is the corresponding diagram for the squark-gluino process with an s-channel quark and diagrams $(c)$ and $(d)$ are the scalar four-point interactions for the squark-squark and squark-antisquark processes, respectively. Note that the $s$-channel gluon annihilation diagram is $P$-wave suppressed for the squark-antisquark case so the only contribution comes from the four-squark vertex $(d)$. Note that we shall assume that the difference of squark and gluino masses is sufficiently large, $\left|m_{\tilde{g}}-m_{\tilde{q}}\right|>M \beta^{2}$, so that annihilation contributions that change the sparticle species (e.g. $\tilde{q} \overline{\tilde{q}} \rightarrow \tilde{g} \tilde{g}$ through $t$-channel quark exchange) do not lead to threshold-enhanced contributions to the cross section.

As an example, we discuss the case of gluino-pair production in detail. In this case it is convenient to identify the operator $\psi^{\prime}$ in the PNRSQCD Lagrangian (2.15) and the EFT matrix element in the matching condition (2.31) with the creation operator of the chargeconjugate gluino field $\psi^{c \dagger}$. The non-relativistic limit of the annihilation contribution to the 
matrix element corresponding to figure $1(a)$ is given by

$$
\begin{aligned}
\left.\frac{1}{4 m_{\tilde{g}}^{2}} i \mathcal{M}\left(\tilde{g}_{1} \tilde{g}_{2} \rightarrow \tilde{g}_{3} \tilde{g}_{4}\right)\right|_{\hat{s}=4 M^{2}} ^{\text {ann }} & =(-i) \frac{g_{s}^{2}}{4 m_{\tilde{g}}^{2}} F_{a_{3} a_{4}}^{a} F_{a_{2} a_{1}}^{a}\left(\eta_{2}^{\dagger} \sigma^{i} \xi_{1}\right)\left(\xi_{3}^{\dagger} \sigma^{i} \eta_{4}\right) \\
& =(-i) \frac{g_{s}^{2}}{4 m_{\tilde{g}}^{2}} 2 N_{c} P_{\{a\}}^{\left(8_{a}\right)} \Pi_{\{\alpha\}}^{S=1} \eta_{\alpha_{2}}^{\dagger} \xi_{\alpha_{1}} \xi_{\alpha_{3}}^{\dagger} \eta_{\alpha_{4}}
\end{aligned}
$$

with the generators of the adjoint representation $F_{a_{1} a_{2}}^{a}=i f^{a_{1} a a_{2}}$ and the two-component particle (antiparticle) spinors $\xi(\eta)$. In the second line, we have introduced the projection operators on the $8_{a}$ colour representation and the spin-triplet,

$$
P_{\{a\}}^{\left(8_{a}\right)}=\frac{1}{N_{c}} F_{a_{3} a_{4}}^{a} F_{a_{2} a_{1}}^{a}, \quad \Pi_{\{\alpha\}}^{S=1}=\frac{1}{2} \sigma_{\alpha_{3} \alpha_{4}}^{i} \sigma_{\alpha_{2} \alpha_{1}}^{i} .
$$

To evaluate the matrix element on the left-hand side of (2.31), note that the property of Majorana fermions in an S-wave state (see e.g. [66])

$$
\left(\psi_{i}^{\dagger} \sigma^{i} \psi_{j}^{c}\right)=-\left(\psi_{j}^{\dagger} \sigma^{i} \psi_{i}^{c}\right)
$$

and the anti-symmetry of the $F_{a_{i} a_{j}}^{a}$ imply that all possible four contractions of the external states with the field operators give an identical contribution to the projection of the matrix element on the $8_{a}, S=1$ state. Taking the factor $1 / 2$ for identical particles in (2.31) into account, the potential is therefore obtained by multiplying the matrix element (2.32) by a symmetry factor of $1 / 2$. The final result for the coefficients in the decomposition (2.19) of the annihilation potential reads

$$
\delta_{\mathrm{ann}} \tilde{V}^{R_{\alpha}, S}=\frac{\pi \alpha_{s}}{m_{\tilde{g}}^{2}}\left[N_{c} \delta_{R_{\alpha}, 8} \delta_{S, 1}\right] .
$$

The corresponding result for quark-antiquark annihilation [67] is obtained by changing the colour factor $N_{c}$ to $T_{F}=\frac{1}{2}$ due to the normalization of the colour projector $P^{(8)}$ in the fundamental representation and multiplying by a factor of two due to the absence of the symmetry factor for Dirac fermions.

The results for the remaining squark and gluino pair-production processes are obtained in a similar way and collected in table 2 in the form of coefficients $A_{\{n\}}^{R_{\alpha}, S}$ defined by

$$
\delta_{\mathrm{ann}} \tilde{V}_{\{n\}}^{R_{\alpha}, S}=\frac{\pi \alpha_{s}}{M^{2}} A_{\{n\}}^{R_{\alpha}, S} .
$$

The flavour labels are only required for the cases of squark-squark and squark-antisquark scattering where the label $n_{i}$ denoting the ten light-flavour squark states is considered as a pair $\left(i, \lambda_{i}\right)$ of a flavour label $i$ and a helicity label $\lambda_{i}=1$ (2) for left (right) squarks. The results are given with a general squark-mass dependence, although we only require the equal-mass case for our numerical results. We have assumed vanishing squark mixing which implies that the matrix $X_{\lambda_{i} \lambda_{j}}^{i}$ appearing in the four-squark vertex [68] has the form

$$
X_{\lambda_{i} \lambda_{j}}^{i}=(-1)^{\frac{\lambda_{i}+\lambda_{j}}{2}} \delta_{\lambda_{i} \lambda_{j}} \delta_{i j}
$$




\begin{tabular}{|l|c|}
\hline$\left(\tilde{s} \tilde{s}^{\prime}\right)_{S}^{R_{\alpha}}$ & $A_{\{n\}}^{R_{\alpha}, S}$ \\
\hline$(\tilde{q} \overline{\tilde{q}})^{1}$ & $-D_{1} \frac{M}{2 m_{\mathrm{red}}} X_{\lambda_{3} \lambda_{1}}^{i_{1}} X_{\lambda_{2} \lambda_{4}}^{i_{2}}$ \\
$(\tilde{q} \overline{\tilde{q}})^{8}$ & $\frac{M}{2 m_{\mathrm{red}}}\left(T_{F} X_{\lambda_{1} \lambda_{2}}^{i_{1}} X_{\lambda_{3} \lambda_{4}}^{i_{3}}-D_{8} X_{\lambda_{3} \lambda_{1}}^{i_{1}} X_{\lambda_{2} \lambda_{4}}^{i_{2}}\right)$ \\
\hline$(\tilde{q} \tilde{q})^{\overline{3}}$ & $D_{\overline{3}} T_{F} \frac{M}{2 m_{\mathrm{red}}}\left(X_{\lambda_{1} \lambda_{3}}^{i_{1}} X_{\lambda_{2} \lambda_{4}}^{i_{2}}-X_{\lambda_{1} \lambda_{4}}^{i_{1}} X_{\lambda_{2} \lambda_{3}}^{i_{2}}\right)$ \\
$(\tilde{q} \tilde{q})^{6}$ & $D_{6} T_{F} \frac{M}{2 m_{\mathrm{red}}}\left(X_{\lambda_{1} \lambda_{3}}^{i_{1}} X_{\lambda_{2} \lambda_{4}}^{i_{2}}+X_{\lambda_{1} \lambda_{4}}^{i_{1}} X_{\lambda_{2} \lambda_{3}}^{i_{2}}\right)$ \\
\hline$(\tilde{q} \tilde{g})_{\frac{1}{2}}^{3}$ & $C_{F} \frac{m_{\tilde{q}}+m_{\tilde{g}}}{2 m_{\tilde{q}}}$ \\
\hline$(\tilde{g} \tilde{g})_{1}^{8}$ & $N_{c}$ \\
\hline
\end{tabular}

Table 2. Non-vanishing values for the coefficients $A_{\{n\}}^{R_{\alpha}, S}$ of the annihilation potential (2.36) for the different squark and gluino production processes for the colour state $R_{\alpha}$ and spin state $S$ (if applicable). The helicity labels for the squark $i$ are denoted by $\lambda_{i}$. The matrix appearing in the squark potentials is defined in eq. (2.37).

Since the annihilation potential (2.36) is momentum independent (proportional to $\delta^{(3)}(z)$ in coordinate space), the resulting Green function correction (2.23) is proportional to the square of the Coulomb Green function at the origin,

$$
\delta_{\mathrm{ann}} G_{R_{\alpha},\{n\}}^{(1) S}=-\frac{\pi \alpha_{s}}{M^{2}} A_{\{n\}}^{R_{\alpha}, S}\left[G_{R_{\alpha}}^{(0)}(0,0 ; E)\right]^{2} .
$$

Note that the squark-squark annihilation potential in table 2 shares the (anti-)symmetry with respect to the exchange of initial- or final-state flavours of the potential function in the $\overline{3}$ (6) colour channel, as assumed in (2.23).

The correction to the potential function $J_{R_{\alpha}}^{S}$ is given by twice the imaginary part of $\delta G_{R_{\alpha}}^{S}$,

$$
\delta_{\mathrm{ann}} J_{R_{\alpha},\{n\}}^{S}=-\frac{4 \pi \alpha_{s}}{M^{2}} A_{\{n\}}^{R_{\alpha}, S} \operatorname{Re}\left[G_{R_{\alpha}}^{(0)}(0,0 ; E)\right] \operatorname{Im}\left[G_{R_{\alpha}}^{(0)}(0,0 ; E)\right],
$$

with

$$
\begin{aligned}
& \operatorname{Re}\left[G_{R_{\alpha}}^{(0)}(0,0 ; E)\right]=\frac{m_{\mathrm{red}}^{2} D_{R_{\alpha}} \alpha_{s}}{\pi} \ln \beta+\ldots, \\
& \operatorname{Im}\left[G_{R_{\alpha}}^{(0)}(0,0 ; E)\right]=\frac{\beta}{4 \pi} \frac{\left(m_{\tilde{s}} m_{\tilde{s}^{\prime}}\right)^{3 / 2}}{M}+\mathcal{O}\left(\alpha_{s}\right),
\end{aligned}
$$

where the ellipsis denotes non-logarithmic terms or terms of higher order in $\alpha_{s}$.

To obtain the annihilation correction to the cross section from the factorization formula (2.9), the non-trivial flavour structure of the hard production process must be taken into account by introducing a flavour dependent hard function, $H_{i,\{n\}}^{S}$. This is schematically of the form $H_{i,\{n\}}^{S} \sim C_{i, n_{1} n_{2}}^{S} C_{i, n_{3} n_{4}}^{S *}$, where the matching coefficient $C_{i, n_{i} n_{j}}^{S}$ is related 


\begin{tabular}{|l|c|}
\hline$p p^{\prime} \rightarrow\left(\tilde{s} \tilde{s}^{\prime}\right)_{S}^{R_{\alpha}}$ & $\nu_{\text {ann }}^{R_{\alpha}, S}$ \\
\hline$g g \rightarrow(\tilde{q} \tilde{\tilde{q}})^{R_{\alpha}}$ & $-D_{R_{\alpha}} \frac{M}{2 m_{\text {red }}}$ \\
$q \bar{q} \rightarrow(\tilde{q} \tilde{\tilde{q}})^{R_{\alpha}}$ & $D_{R_{\alpha}} \frac{M}{2 m_{\text {red }}}$ \\
\hline$q_{i} q_{j} \rightarrow\left(\tilde{q}_{i} \tilde{q}_{j}\right)^{R_{\alpha}}$ & $2 T_{F} D_{R_{\alpha}} \frac{M}{2 m_{\mathrm{red}}}$ \\
\hline$q g \rightarrow(\tilde{q} \tilde{g})_{\frac{1}{2}}^{3}$ & $C_{F} \frac{m_{\tilde{q}}+m_{\tilde{g}}}{2 m_{\tilde{q}}}$ \\
\hline$q \bar{q} \rightarrow(\tilde{g} \tilde{g})_{1}^{8_{a}}$ & $N_{c}$ \\
\hline
\end{tabular}

Table 3. Non-vanishing values of the coefficient $\nu_{\mathrm{ann}}^{R_{\alpha}, S}$ of the annihilation corrections for the different squark and gluino production processes for the colour state $R_{\alpha}$ and spin state $S$ (if applicable).

to the amputated production amplitude of the sparticle state $\left(\tilde{s}_{n_{i}} \tilde{s}_{n_{j}}\right)_{S}^{R_{\alpha}}$ at threshold (see eqs. (2.60) and (3.10) in [12]). The product of the flavour-dependent hard function and the annihilation contribution to the potential function therefore takes the interference of the different production channels $\tilde{s}_{n_{1}} \tilde{s}_{n_{2}}$ and $\tilde{s}_{n_{3}} \tilde{s}_{n_{4}}$ into account, which are connected by a rescattering through the annihilation potential $\delta_{\text {ann }} \tilde{V}_{\{n\}}$. The potential function $J_{R_{\alpha}}^{S}(E)$ appearing in (2.9) is the flavour-averaged potential function defined by

$$
J_{R_{\alpha}}^{S}(E)=\frac{J_{R_{\alpha},\{n\}}^{S}(E) H_{i,\{n\}}^{S(0)}}{H_{i}^{S(0)}},
$$

where $H_{i}^{(0) S}$ is the flavour-summed LO hard function that appears in the LO cross section (2.13).

The NNLO annihilation correction to the cross section in the colour and spin state $R_{\alpha}$ and $S$ is then given by

$$
\begin{aligned}
\Delta \hat{\sigma}_{p p^{\prime}, \text { ann }}^{(2) R_{\alpha}, S}\left(\hat{s}, \mu_{f}\right) & =\left.H_{i}^{S(0)} \delta_{\text {ann }} J_{R_{\alpha}}^{S}(E)\right|_{\mathcal{O}\left(\alpha_{s}^{2}\right)} \\
& =\hat{\sigma}_{p p^{\prime}}^{(0) R_{\alpha}, S}(\hat{s}) \alpha_{s}^{2} \ln \beta\left(-\frac{D_{R_{\alpha}}}{2} \frac{4 m_{\mathrm{red}}^{2}}{M^{2}} \nu_{\text {ann }}^{R_{\alpha}, S}\right),
\end{aligned}
$$

where it was used that only the leading-order soft function contributes to the $\mathcal{O}\left(\alpha_{s}^{2} \ln \beta\right)$ correction, which renders the convolution in (2.9) trivial. The annihilation correction relative to the LO cross section has been defined in terms of the coefficient

$$
\nu_{\mathrm{ann}}^{R_{\alpha}, S}=\frac{A_{\{n\}}^{R_{\alpha}, S} H_{i,\{n\}}^{S(0)}}{H_{i}^{S(0)}},
$$

whose values for all squark and gluino pair-production processes are collected in table 3 .

The results for the squark-antisquark production process can be derived from the potential coefficients given in table 2 and the definition (2.44) using the fact that, in 
the absence of flavour violation, the matching coefficients for the gluon initial state are non-vanishing only for equal squark flavours and helicity labels, $g g \rightarrow \tilde{q}_{i \lambda_{i}} \overline{\tilde{q}}_{i \lambda_{i}}$. For the quark-antiquark initial state only opposite helicity labels contribute, while the flavours are fixed by the initial-state quarks, $q_{i} \bar{q}_{j} \rightarrow \tilde{q}_{i L} \overline{\tilde{q}}_{j R}$ and $q_{i} \bar{q}_{j} \rightarrow \tilde{q}_{i R} \overline{\tilde{q}}_{j L}$ (see e.g. ref. [12]). For squark-squark production, the helicity labels of the two squarks agree, $q_{i} q_{j} \rightarrow \tilde{q}_{i L} \tilde{q}_{j L}$ and $q_{i} q_{j} \rightarrow \tilde{q}_{i R} \tilde{q}_{j R}$, while the symmetry properties of the matching coefficients under exchange of the squarks follow from the respective colour representation, i.e. the coefficients for the $\overline{3}(6)$ state are anti-symmetric (symmetric).

For the example of gluino pair-production we obtain the correction

$$
\Delta \hat{\sigma}_{q \bar{q}, \text { ann }}^{(2) R_{\alpha}=8, S=1}(\hat{s})=\hat{\sigma}_{q \bar{q}}^{(0) R_{\alpha}=8}(\hat{s}) \alpha_{s}^{2} \ln \beta\left(-\frac{D_{8} N_{c}}{2}\right),
$$

which agrees with the one for top-quark pair production in eq. (4.15) of [54] after the appropriate changes of the group-theory factors, $N_{c} \rightarrow T_{F}$ and $D_{8} \rightarrow \frac{1}{2}\left(C_{A}-2 C_{F}\right)$, and multiplication by a factor of 2 due to the Dirac nature of the top quark.

\subsubsection{NLO potential function}

For resummation at NNLL accuracy, the NLO potential function has to be inserted into the resummation formula (2.10). It is given by the perturbative solution (2.23) of the Schrödinger equation with the potential (2.16) and can be written in the form

$$
J_{R_{\alpha}}^{S}(E)=2 \operatorname{Im}\left[G_{R_{\alpha}}^{(0)}(0,0 ; E) \Delta_{\mathrm{nC}}^{R_{\alpha}, S}(E)+G_{R_{\alpha}}^{(1)}(0,0 ; E)\right] .
$$

The function $G_{R_{\alpha}}^{(1)}$ is obtained from one insertion of the NLO Coulomb potential and includes all terms of the form $\alpha_{s} \times\left(\alpha_{s} / \beta\right)^{n}$. Its explicit expression can be obtained by the simple replacement $m_{t} \rightarrow 2 m_{\text {red }}$ from the corresponding result for top-pair production quoted in eq. (A.1) of [51]. The factor $\Delta_{\mathrm{nC}}^{R_{\alpha}, S}$ arises from an insertion of the one-loop $1 / \mathrm{m}$, the tree-level spin-dependent non-Coulomb, and the annihilation potentials. It is given in terms of the results of sections 2.3 .1 and 2.3.2 as

$$
\Delta_{\mathrm{nC}}^{R_{\alpha}, S}(E)=1+\alpha_{s}^{2}\left(\mu_{C}\right) \ln \beta\left[C_{A} D_{R_{\alpha}}-2 D_{R_{\alpha}}^{2}\left(1+\nu_{\mathrm{spin}}^{S}\right)-\frac{D_{R_{\alpha}}}{2} \frac{4 m_{\mathrm{red}}^{2}}{M^{2}} \nu_{\mathrm{ann}}^{R_{\alpha}, S}\right] \theta(E) .
$$

Eq. (2.46) combines the non-Coulomb correction (2.47) with all-order Coulomb resummation and therefore includes corrections of the form $\alpha_{s}^{2} \ln \beta \times\left(\alpha_{s} / \beta\right)^{n}$. As in [51] we do not resum the logarithms arising from the non-Coulomb corrections, which formally are also an NNLL contribution. Such a resummation can in principle be performed using renormalization group methods in PNR(S)QCD, but is left for future work.

\subsubsection{Bound-state effects}

In the colour channels with an attractive Coulomb potential $\left(D_{R_{\alpha}}<0\right)$, the Coulomb Green function develops bound-state poles below threshold,

$$
J_{R_{\alpha}}(E)=2 \sum_{n=1}^{\infty} \delta\left(E-E_{n}\right) R_{n} \theta\left(-D_{R_{\alpha}}\right), \quad E<0
$$


with binding energies

$$
E_{n}=-\frac{2 m_{\mathrm{red}} \alpha_{s}^{2} D_{R_{\alpha}}^{2}}{4 n^{2}}\left(1+\frac{\alpha_{s}}{4 \pi} e_{1}\right)
$$

and residues

$$
R_{n}=\left(\frac{2 m_{\mathrm{red}}\left(-D_{R_{\alpha}}\right) \alpha_{s}}{2 n}\right)^{3}\left(1+\frac{\alpha_{s}}{4 \pi} \delta r_{1}\right)
$$

The values of the NLO corrections $e_{1}$ and $\delta r_{1}[69,70]$ are quoted in [51], where again the replacement $m_{t} \rightarrow 2 m_{\text {red }}$ is implied.

For long-lived squarks or gluinos, the poles correspond to physical gluinonium or squarkonium bound states, which subsequently decay to di-photon or di-jet final states. In this paper we do not consider this case with the resulting very different collider signals compared to the usual missing-energy signatures. For squarks and gluinos that decay within the LHC detectors, the bound-state poles are smeared out by the finite decay widths. The resulting contribution to the total cross section from partonic centre-of-mass energies below the nominal production threshold can be included in the resummation formula (2.10) by using the bound-state contributions (2.48) for vanishing decay widths. The convolution of these corrections with the soft corrections is performed as described in [51]. In [44] this procedure has been compared at NLL accuracy to the description of finite-width effects through a complex energy $E \rightarrow E+i\left(\Gamma_{\tilde{s}}+\Gamma_{\tilde{s}^{\prime}}\right) / 2$ in the potential function. It was found that finite width effects on the NLL $K$-factors for squark-squark and squarkantisquark production processes are well below the $5 \%$ level while they can become of the order of $10 \%$ or even larger for gluino production processes with a gluino decay width above $\Gamma_{\tilde{g}} / m_{\tilde{g}} \gtrsim 5 \%$. However, this case only occurs for SQCD two-body decays $\tilde{g} \rightarrow \tilde{q} q$ in the region $m_{\tilde{g}} \gtrsim 1.3 m_{\tilde{q}}$, where gluino production is kinematically suppressed and gives a small contribution to the total SUSY production rate. In phenomenologically relevant parameter-space regions of the MSSM, the finite width effects are therefore smaller than the remaining perturbative uncertainty of the NNLL calculation, which justifies the use of the narrow-width approximation in this paper.

\subsubsection{Fixed-order treatment of Coulomb corrections}

In order to assess the impact of Coulomb resummation and to compare to NNLL predictions treating Coulomb corrections at fixed order, we also consider an approximation $\mathrm{NNLL}_{\text {fixed-C }}$ where the product of hard and Coulomb corrections in the resummation formula (2.10) is replaced by its fixed-order expansion up to $\mathcal{O}\left(\alpha_{s}^{2}\right)$,

$$
H_{i}\left(\mu_{h}\right) J_{R_{\alpha}}^{S}(E) \Rightarrow H_{i}^{(0)}\left(\mu_{h}\right) \frac{\beta}{2 \pi} \frac{\left(m_{\tilde{s}} m_{\tilde{s}^{\prime}}\right)^{3 / 2}}{M} \Delta_{\mathrm{hC}}^{\mathrm{NNLO}}\left(\hat{s}, \mu_{h}, \mu_{f}\right)
$$


The correction factor is given by

$$
\begin{aligned}
\Delta_{\mathrm{hC}}^{\mathrm{NNLO}}\left(\hat{s}, \mu_{h}, \mu_{f}\right)= & \left\{\left(1-\alpha_{s}\left(\mu_{f}\right) \frac{\pi D_{R_{\alpha}}}{2} \sqrt{\frac{2 m_{\mathrm{red}}}{E}}\right)\left(1+\frac{\alpha_{s}\left(\mu_{h}\right)}{4 \pi} h_{i}^{(1)}\left(\mu_{h}\right)\right)\right. \\
& +\alpha_{s}^{2}\left(\mu_{f}\right)\left[\frac{\pi^{2} D_{R_{\alpha}}^{2}}{12}\left(\frac{2 m_{\mathrm{red}}}{E}\right)+\frac{D_{R_{\alpha}}}{8} \sqrt{\frac{2 m_{\mathrm{red}}}{E}}\left(\beta_{0} \ln \left(\frac{8 m_{\mathrm{red}} E}{\mu_{f}^{2}}\right)-a_{1}\right)\right. \\
& \left.\left.+\frac{1}{2} \ln \frac{E}{M}\left(C_{A} D_{R_{\alpha}}-2 D_{R_{\alpha}}^{2}\left(1+\nu_{\mathrm{spin}}^{S}\right)-\frac{D_{R_{\alpha}}}{2} \frac{4 m_{\mathrm{red}}^{2}}{M^{2}} \nu_{\mathrm{ann}}^{R_{\alpha}, S}\right)\right]\right\} .
\end{aligned}
$$

In this approximation one can derive an analytic formula for the NNLL cross-section that is given in appendix A.2.

\subsubsection{Numerical size of non-Coulomb potential, annihilation and bound state contributions}

In table 4 we illustrate the numerical impact of the non-Coulomb and annihilation potentials computed in sections 2.3.1 and 2.3.2, respectively, as well as the bound-state corrections discussed in section 2.3.4. The correction to the full NNLL cross section is obtained by removing the respective contribution to the NLO potential function (2.46) from the resummation formula (2.10). In table 4 the results for a given partonic initial state are normalized to the total NLO cross section, so that they specify the contribution to the NNLL K-factor defined in (3.2) below. The choices of the input parameters, PDFs and the various scales are discussed in sections 2.4 and 3.1 .

The corrections from the bound-state contributions and the non-Coulomb potential are of comparable size and generally in the per-cent range. The size of the potential corrections for the different processes follows a similar pattern as the full NNLL corrections discussed in section 3. The largest values are observed for gluino-pair production, where they grow above $10 \%$ at high masses. In contrast, for squark-squark production all types of corrections stay below a percent. The annihilation correction is typically an order of magnitude smaller than the two other types of corrections and therefore phenomenologically negligible. For the case of squark-antisquark and gluino-pair production, the size of the corrections from the quark-antiquark and gluon initial states reflects the relative contribution of these partonic channels to the total cross section. For gluino-pair production, the quark-antiquark channel contribution to the numbers in table 4 is further suppressed, since only a single colour channel contributes to S-wave production at threshold, cf. table 1.

\subsection{Scale choices}

The resummed cross section (2.10) depends on the factorization scale $\mu_{f}$, which we set to $\mu_{f}=M$ as a default, the soft scale $\mu_{s}$, the hard scale $\mu_{h}$, as well as on the scale $\mu_{C}$ used in the Coulomb function. While the solution to the renormalization group equations for the hard and soft functions is formally independent on $\mu_{h}$ and $\mu_{s}$, due to the truncation of the perturbative series the NNLL resummed cross section contains a residual dependence on these scales at $\mathcal{O}\left(\alpha_{s}^{2}\right)$. We specify our scale choices here which, with the exception of the soft scale, follow the treatment in $[16,51]$. 


\begin{tabular}{|c|c||l|l|r||r|r|c|}
\hline \multicolumn{2}{|l||}{} & \multicolumn{3}{c||}{$m_{\tilde{q}}=1 \mathrm{TeV}, m_{\tilde{g}}=1.5 \mathrm{TeV}$} & \multicolumn{3}{c|}{$m_{\tilde{q}}=2.5 \mathrm{TeV}, m_{\tilde{g}}=3 \mathrm{TeV}$} \\
\hline$\tilde{s} \tilde{s}^{\prime}$ & $p p^{\prime}$ & $\delta_{\mathrm{BS}}$ & $\delta_{\mathrm{nC}}$ & \multicolumn{1}{c|}{$\delta_{\mathrm{ann}}$} & $\delta_{\mathrm{BS}}$ & $\delta_{\mathrm{nC}}$ & $\delta_{\mathrm{ann}}$ \\
\hline$\tilde{q} \tilde{\tilde{q}}$ & $q \bar{q}$ & $1.5 \%$ & $3.2 \%$ & $0.4 \%$ & $4.2 \%$ & $5.7 \%$ & $0.8 \%$ \\
& $g g$ & $0.3 \%$ & $0.8 \%$ & $-0.1 \%$ & $0.4 \%$ & $0.6 \%$ & $-0.1 \%$ \\
\hline$\tilde{q}_{i} \tilde{q}_{j}$ & $q q$ & $0.1 \%$ & $0.5 \%$ & $0.1 \%$ & $0.2 \%$ & $0.4 \%$ & $0.1 \%$ \\
\hline$\tilde{q} \tilde{g}$ & $q g$ & $0.8 \%$ & $1.7 \%$ & $-0.3 \%$ & $2.4 \%$ & $3.3 \%$ & $-0.5 \%$ \\
\hline$\tilde{g} \tilde{g}$ & $q \bar{q}$ & $0.2 \%$ & $0.3 \%$ & $-0.1 \%$ & $-0.1 \%$ & $-0.1 \%$ & $0.03 \%$ \\
& $g g$ & $5.1 \%$ & $8.5 \%$ & - & $14.9 \%$ & $16.2 \%$ & - \\
\hline
\end{tabular}

\begin{tabular}{|c|c||l|l|r||r|r|r|}
\hline \multicolumn{2}{|l||}{} & \multicolumn{3}{c||}{$m_{\tilde{q}}=1.5 \mathrm{TeV}, m_{\tilde{g}}=1 \mathrm{TeV}$} & \multicolumn{3}{c|}{$m_{\tilde{q}}=3 \mathrm{TeV}, m_{\tilde{g}}=2.5 \mathrm{TeV}$} \\
\hline$\tilde{s} \tilde{s}^{\prime}$ & $p p^{\prime}$ & $\delta_{\mathrm{BS}}$ & $\delta_{\mathrm{nC}}$ & $\delta_{\mathrm{ann}}$ & $\delta_{\mathrm{BS}}$ & $\delta_{\mathrm{nC}}$ & $\delta_{\mathrm{ann}}$ \\
\hline$\tilde{q} \overline{\tilde{q}}$ & $q \bar{q}$ & $1.8 \%$ & $3.6 \%$ & $0.5 \%$ & $5.1 \%$ & $6.2 \%$ & $0.8 \%$ \\
& $g g$ & $0.2 \%$ & $0.4 \%$ & $-0.1 \%$ & $0.4 \%$ & $0.5 \%$ & $-0.1 \%$ \\
\hline$\tilde{q}_{\tilde{q}} \tilde{q}_{j}$ & $q q$ & $0.1 \%$ & $0.3 \%$ & $0.1 \%$ & $0.3 \%$ & $0.4 \%$ & $0.1 \%$ \\
\hline$\tilde{q} \tilde{g}$ & $q g$ & $1.5 \%$ & $2.8 \%$ & $-0.3 \%$ & $3.2 \%$ & $4.0 \%$ & $-0.5 \%$ \\
\hline$\tilde{g} \tilde{g}$ & $q \bar{q}$ & $0.3 \%$ & $0.4 \%$ & $-0.1 \%$ & $0.6 \%$ & $0.6 \%$ & $-0.2 \%$ \\
& $g g$ & $4.0 \%$ & $7.8 \%$ & - & $10.5 \%$ & $13.3 \%$ & - \\
\hline
\end{tabular}

Table 4. Contribution of the bound-state, non-Coulomb and annihilation corrections to the NNLL result for the LHC with $\sqrt{s}=13 \mathrm{TeV}$. The corrections are normalized to the total NLO cross sections.

Hard scale. Our default value for the hard scale is $\mu_{h}=2 M$ which can be motivated by the logarithmic structure of the renormalization group equation of the hard function [50]. This choice is also seen to eliminate the logarithms in the expression of the hard function (2.14). To estimate the theoretical uncertainty from the choice of the hard scale, we include a variation in the interval $M \leq \mu_{h} \leq 4 M$ in our estimate of the theoretical uncertainties.

Soft scale. A resummation of all logarithms of $\beta$ in the partonic cross section could be achieved by the running soft scale $\mu_{s} \sim M \beta^{2}$ which, however, renders the convolution of the partonic cross-section (2.10) with the PDFs unintegrable and leads to a Landau pole in $\alpha_{s}\left(\mu_{s}\right)$. Instead, ref. [60] proposed a fixed soft scale that minimizes the one-loop soft corrections to the hadronic cross section. Alternatively, a running soft scale $\mu_{s}=$ $k_{s} M \operatorname{Max}\left[\beta^{2}, \beta_{\text {cut }}^{2}\right]$ was introduced in [51]. This method was applied to squark and gluino production at NLL accuracy in [16] with $k_{s}=1$ and choosing the parameter $\beta_{\text {cut }}$ small enough to justify the resummation of logarithms $\ln \beta_{\text {cut }}$ in the lower interval, and large enough so that threshold logarithms can be treated perturbatively in the upper interval, following the procedure of [51].

Recently, Sterman and Zeng [55] considered an expansion of the logarithm of the parton luminosity function,

$$
\ln L_{p p^{\prime}}\left(\tau_{0} / z, \mu\right)=s_{p p^{\prime}}^{(0)}\left(\tau_{0}, \mu\right)+s_{p p^{\prime}}^{(1)}\left(\tau_{0}, \mu\right) \ln z+\ldots
$$


with

$$
s_{p p^{\prime}}^{(1)}\left(\tau_{0}, \mu\right)=-\left.\frac{d \ln L_{p p^{\prime}}(\tau, \mu)}{d \ln \tau}\right|_{\tau=\tau_{0}} .
$$

To the extent that the convolution of the parton luminosity with the partonic cross section is dominated by the $\ln z$ term, it was shown in [55] that the momentum-space resummation method is equivalent to the traditional resummation in Mellin-moment space if the soft scale is chosen as

$$
\mu_{s}=\frac{2 M e^{-\gamma_{E}}}{s_{p p^{\prime}}^{(1)}} .
$$

Note that the scale choice (2.55) amounts to the use of a different soft scale in every partonic channel. As demonstrated in [55] for the case of Higgs production, the singlepower approximation of the parton luminosity provides a dominant contribution to the convolution with the partonic cross section, so the scale choice (2.55) is motivated also for the use of the exact PDFs. This conclusion is also supported by an analysis using the saddle-point approximation [71]. We therefore adopt (2.55) as our default choice for the soft scale. This choice is also convenient for the numerical implementation, since it can be determined during the evaluation of the cross section at very small computational cost without a prior minimization procedure as for the other scale-setting procedures. In our implementation, the flavour-summed parton luminosities mentioned below (2.5) are used for the determination of the soft scale in the case of initial-state quarks. The theoretical uncertainty due to the scale choice is estimated by varying $\mu_{s}$ from one-half to twice the default scale. Note that we keep the factorization scale fixed in the determination of the soft scale, i.e. we always use the default value $\mu_{f}=M$ in (2.54).

Coulomb scale. At NNLL accuracy the scale $\mu$ in the potential function (2.46) can be chosen independently of the other scales. NLL effects related to Coulomb exchange can be resummed by choosing the scale of the order of $\sqrt{2 m_{\text {red }} M} \beta$, which is the typical virtuality of Coulomb gluons. For small $\beta$, in production channels with an attractive Coulomb potential the relevant scale is instead given by the Bohr scale $2 m_{\text {red }}\left|D_{R_{\alpha}}\right| \alpha_{s}$ set by the first bound state, as can be seen from the $\beta \rightarrow 0$ limit of the NLO potential function quoted in [51]. We thus choose the scale in $J_{R_{\alpha}}$ to be

$$
\mu_{C}=\operatorname{Max}\left\{2 \alpha_{s}\left(\mu_{C}\right) m_{\text {red }}\left|D_{R_{\alpha}}\right|, 2 \sqrt{2 m_{\text {red }} M} \beta\right\}
$$

Note that no bound states arise for a repulsive potential, $D_{R_{\alpha}}>0$, in which case $J_{R_{\alpha}}$ vanishes for small $\beta$. Therefore the above argument does not determine the Coulomb scale for $\beta \rightarrow 0$ in this case. We nevertheless use the prescription (2.56) also for a repulsive potential where resummation of Coulomb corrections leads to small effects, so that the precise choice of $\mu_{C}$ has a negligible numerical impact on predictions of the cross section. We vary $\mu_{C}$ from one-half to twice the default value (2.56) to estimate the theoretical uncertainty. 


\section{Numerical results}

\subsection{Setup}

In order to include the known fixed-order NLO corrections without kinematic approximation, we match the NNLL-resummed result to the NLO result from PROSPINO, supplemented with the threshold approximation of the NNLO cross sections, where all constant contributions at $\mathcal{O}\left(\alpha_{s}^{2}\right)$ are set to zero. In order to avoid double counting, the fixed-order expansion of the NNLL corrections up to $\mathcal{O}\left(\alpha_{s}^{2}\right)$ is subtracted from the cross section. Our matched predictions are therefore given by

$$
\hat{\sigma}_{p p^{\prime} \text { matched }}^{\mathrm{NNLL}}(\hat{s})=\left[\hat{\sigma}_{p p^{\prime}}^{\mathrm{NNL}}(\hat{s})-\hat{\sigma}_{p p^{\prime}}^{\mathrm{NNLL}(2)}(\hat{s})\right]+\hat{\sigma}_{p p^{\prime}}^{\mathrm{NLO}}(\hat{s})+\hat{\sigma}_{\mathrm{app}, p p^{\prime}}^{\mathrm{NNLO}}(\hat{s}) .
$$

The approximate NNLO cross section is obtained from eq. (A.1) in [53] by inserting the one-loop hard functions $2 \operatorname{Re}\left(C_{X}\right)=h_{1}^{(i)}$, the results for $\nu_{\text {spin }}^{S}$ given in (2.29) and adding the annihilation contribution (2.43). The expansion of the NNLL correction to $\mathcal{O}\left(\alpha_{s}^{2}\right)$, $\hat{\sigma}_{p p^{\prime}}^{\mathrm{NNLL}(2)}$, is given in appendix A.1. The partonic cross sections in (3.1) are convoluted with the parton luminosities determined using the PDF4LHC15_nnlo_30 PDFs [72], ${ }^{4}$ which combine the MMHT14 [73], CT14 [74] and NNPDF3.0 [75] sets according to [76-78]. We have used the ManeParse [79] interface for some calculations. In the hard functions we use $m_{t}=173.2 \mathrm{GeV}$.

In our results we include an estimate of the theoretical uncertainties of our default implementation from various sources. ${ }^{5}$

Factorization scale uncertainty: the factorization scale $\mu_{f}$ is varied between half and twice the default value, i.e. $M / 2<\mu_{f}<2 M$. For the resummed result, this is done keeping the other scales $\mu_{h}, \mu_{C}$ and $\mu_{s}$ fixed. Note that in the fixed-order results we identify the factorization and renormalization scales, which is the default procedure implemented in the numerical code PROSPINO used for the computation of the fixed-order NLO result [6].

Resummation uncertainty: the soft, hard and Coulomb scale are separately varied around their default values as discussed in section 2.4. Power-suppressed terms are estimated by using the expression $E=\sqrt{\hat{s}}-2 M$ in the argument of the potential function instead of the non-relativistic limit $E=M \beta^{2}$. The resulting uncertainties from all sources are added in quadrature.

Missing higher-order corrections: in order to estimate the uncertainty from uncalculated NNLO corrections we follow [51] and vary the unknown two-loop constant

\footnotetext{
${ }^{4}$ The PDF4LHC15_nnlo_mc set recommended for predictions for the search for new physics [72] yields unphysical negative cross sections at large sparticle masses for some member PDFs. We have checked that the central value and the PDF uncertainty of the PDF4LHC15_nnlo_30 set are in good agreement with the $68 \%$ confidence level predictions of the $\mathrm{MC}$ set, with differences of the central prediction in general below $1 \%$ and staying below $10 \%$ for gluino pair production at the highest considered masses.

${ }^{5}$ The terminology used here follows [16] and differs slightly from the one for $t \bar{t}$ production in [51] where the errors from variation of the hard and Coulomb scales, and of the soft scale for the fixed-scale implementation, were included in the scale uncertainty instead of the resummation uncertainty.
} 

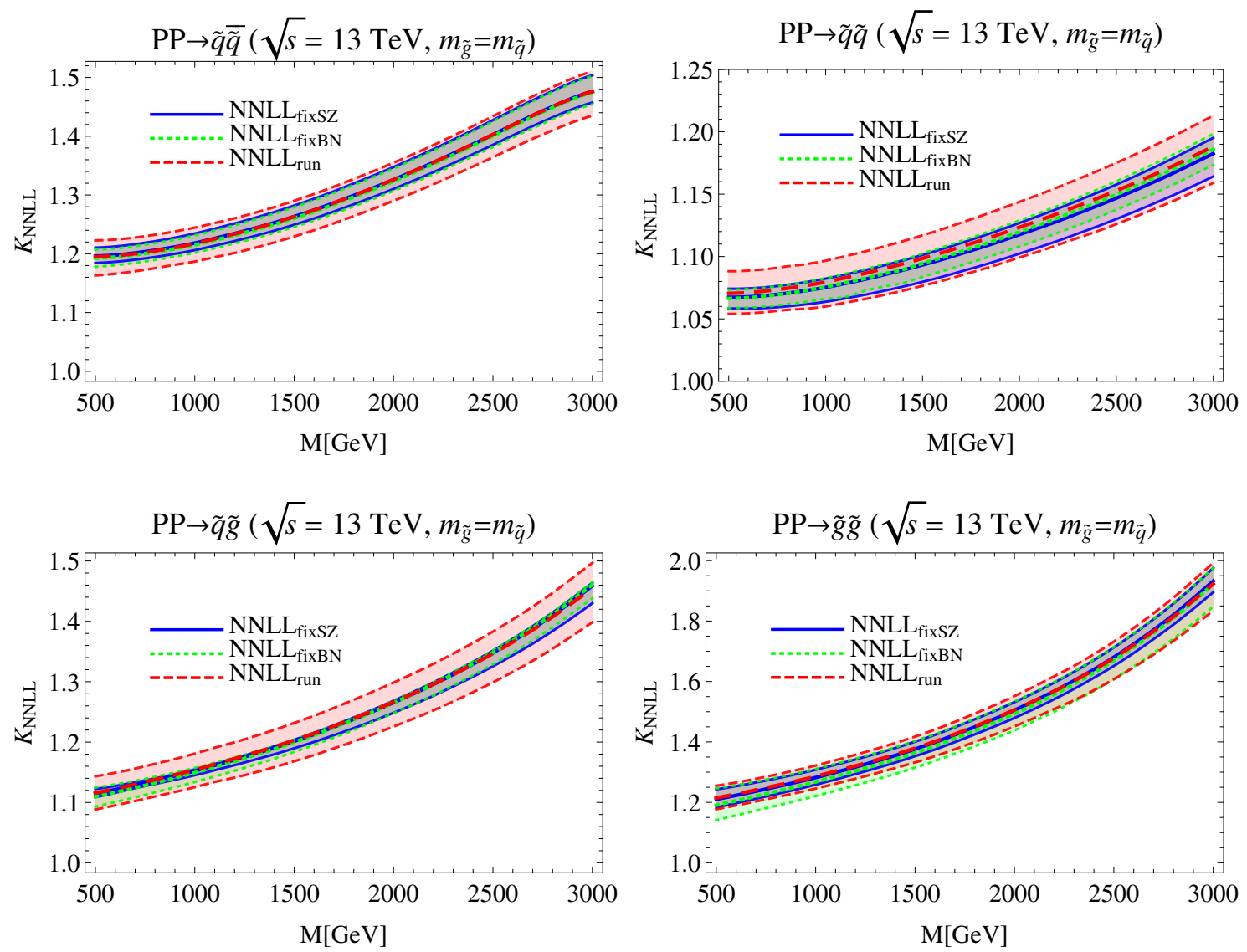

Figure 2. Resummation uncertainty for the NNLL resummed result with the default scale

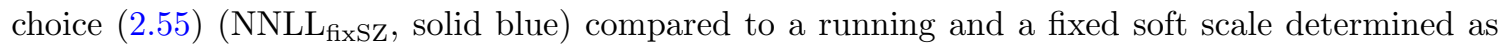
discussed in [16] (NNLL $_{\text {fixBN }}$, short dashed green, and NNLL $_{\text {run }}$, dashed red) for squark-antisquark (top-left), squark-squark (top-right), squark-gluino (bottom-left) and gluino-gluino (bottom-right) at LHC with $\sqrt{s}=13 \mathrm{TeV}$. The central lines represent the $K$-factors for the default scale choice, while the bands give the resummation uncertainties associated with the results. See text for explanation.

term $C_{p p^{\prime}, i}^{(2)}$ in the threshold expansion of the NNLO scaling function $f_{p p^{\prime}, i}^{(2)}$ in (2.8) in the interval $-\left(C_{p p^{\prime}, i}^{(1)}\right)^{2} \leq C_{p p^{\prime}, i}^{(2)} \leq+\left(C_{p p^{\prime}, i}^{(1)}\right)^{2}$, where the one-loop constant $C_{p p^{\prime}, i}^{(1)}$ is defined in (A.4).

PDF $+\boldsymbol{\alpha}_{\boldsymbol{s}}$ uncertainty: we estimate the error due to uncertainties in the PDFs and the strong coupling using the PDF4LHC15_nnlo_30_pdfas set [72], adding the PDF uncertainty at $68 \%$ confidence level in quadrature to the uncertainty from the variation of the strong coupling constant $\alpha_{s}\left(M_{Z}\right)=0.118 \pm 0.0015$.

In the following we will often refer to the sum in quadrature of scale and resummation uncertainty and the variation of the two-loop constant as "total theoretical uncertainty".

In figure 2 we compare the NNLL K-factors, defined in (3.2) below, and the resummation uncertainty for our default implementation with the fixed soft scale (2.55) to an 
implementation using a running scale, as in the previous NLL results in [16], and a fixed scale determined using the method of Becher and Neubert [60]. In the running-scale results the variation of $\mu_{s}$ in the resummation uncertainty is replaced by a variation of the parameters $\beta_{\text {cut }}$ and $k_{s}$ according to the procedure given in [51]. The curves do not include the $C^{(2)}$ error estimate, which is common to all implementations. The central predictions obtained with the different scale-setting methods agree well for all production processes, while the estimate of the resummation uncertainty is larger for the running scale prescription. However, we find that the total theoretical uncertainty including factorization scale variation is similar for the three methods. Note that the agreement of the different scale choices is significantly improved compared to the NLL results (see figure 6 in [16]). Therefore contrary to resummation at NLL level, we find that at NNLL the ambiguity in the choice of the soft scale prescription is negligible with respect to the total theoretical uncertainty.

\subsection{Results}

We present results for squark and gluino production at the LHC for five different higherorder approximations:

- NNLL: the default implementation. Contains the full combined soft and Coulomb resummation, eq. (2.10) including bound-state contributions (2.48) below threshold, matched to $\mathrm{NNLO}_{\text {app }}$ according to (3.1). For the soft scale we adopt the fixed scale given in (2.55).

- NNLL $_{\text {fixed-C: }}$ as above but using the fixed-order NNLO Coulomb terms (2.52) without bound-state effects interfering with resummed soft radiation, and for $\mu_{h}=\mu_{f}$.

- $\mathbf{N N L O}_{\text {app }}$ : the approximate NNLO corrections [53] including the spin-dependent non-Coulomb (2.47) and annihilation terms.

- NLL: the NLL corrections from [16] with combined soft and Coulomb resummation and bound-state effects. Note that the scale choice (2.55) is used for the NLL results as well, whereas a running scale was used as the default in [16].

- $\mathbf{N L L}_{s}$ : soft NLL resummation without Coulomb resummation and for $\mu_{h}=\mu_{f}$.

Our NNLL predictions for the LHC with $\sqrt{s}=13(14 \mathrm{TeV})$ are provided as grids for $m_{\tilde{q}}, m_{\tilde{g}}=200-3000 \mathrm{GeV}(200-3500 \mathrm{GeV})$ [1]. We also provide a Mathematica file with interpolations of the cross sections. As an illustration, our results for the four squark and gluino production processes at the LHC with $\sqrt{s}=13 \mathrm{TeV}^{6}$ for equal squark and gluino masses are shown in figure 3 in the form of K-factors beyond NLO,

$$
K_{\mathrm{X}}=\frac{\sigma_{\mathrm{X}}}{\sigma_{\mathrm{NLO}}} .
$$

\footnotetext{
${ }^{6}$ Results at $\sqrt{s}=8 \mathrm{TeV}$ have been presented in [19] for a slightly different setup using the running soft scale prescription, the MSTW08 PDFs and omitting the annihilation contribution.
} 

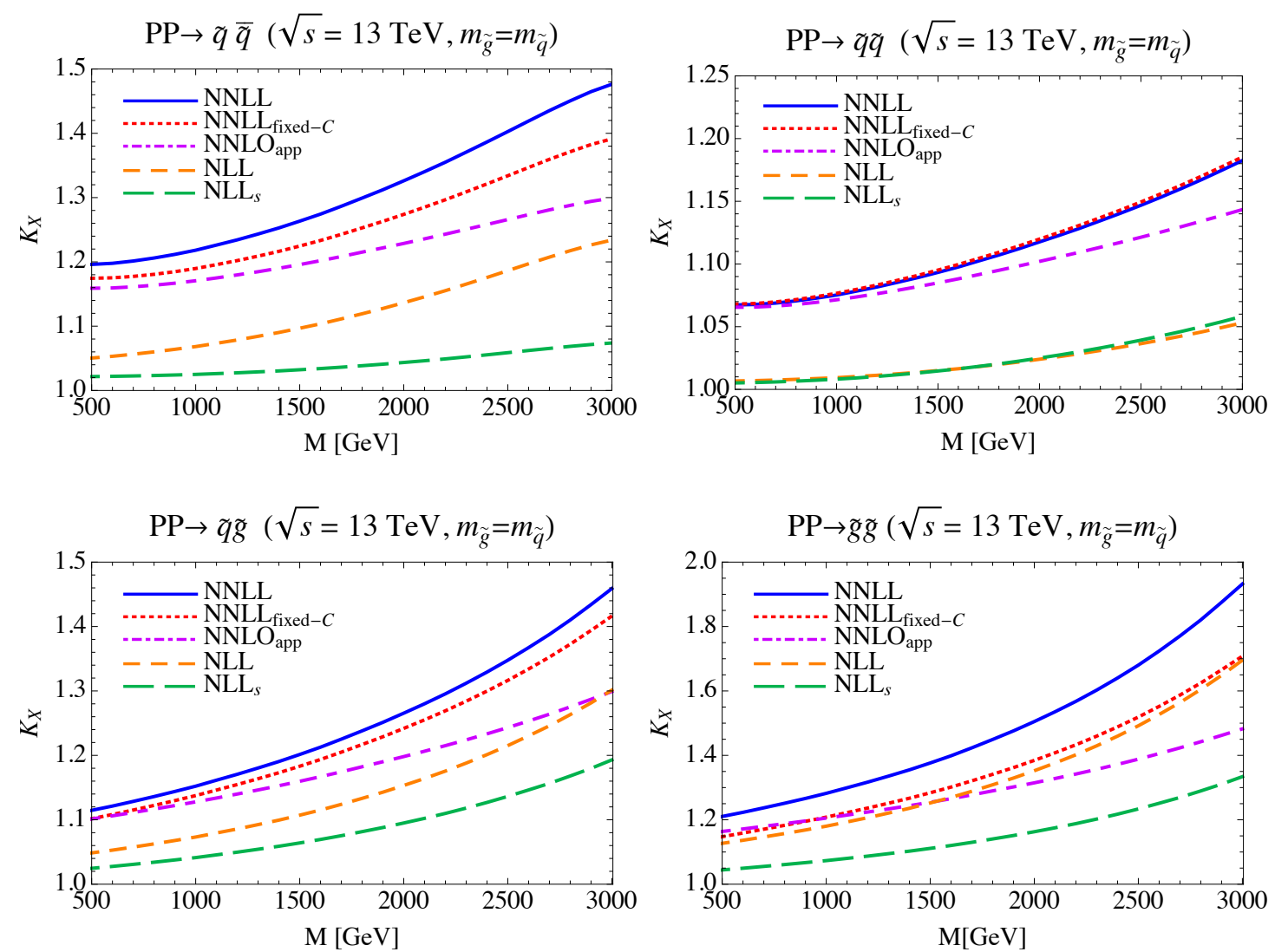

Figure 3. Higher-order corrections relative to the NLO cross section for squark and gluino production at the LHC with $\sqrt{s}=13 \mathrm{TeV}$ for full NNLL resummation (solid blue), NNLL with fixed-order Coulomb corrections (dotted red), approximate NNLO (dot-dashed pink), NLL (dashed orange) and NLL soft (long-dashed green). The PDF4LHC15_nnlo_30 PDFs are used throughout.

Here $X$ denotes one of the approximations NLL, NNLL fixed-C, $_{\text {NNLO }}$ app and NNLL defined above and $\sigma_{\mathrm{NLO}}$ is the fixed-order NLO result obtained using PROSPINO. We use the PDF4LHC15_nnlo PDFs for all results, including the NLO normalization in (3.2), in order to isolate the effects of the higher-order corrections to the partonic cross sections. The corrections relative to NLO can become large for the full NNLL resummation, ranging from up to $18 \%$ for squark-squark production to $90 \%$ for gluino pair production. Compared to the NLL results, the NNLL corrections provide a shift of the cross section by $10-20 \%$ normalized to the NLO prediction. This shows that a stabilization of the perturbative behaviour is achieved by the resummation, in particular for the processes with large corrections such as gluino-pair production. Note that this is only the case for the joint soft-Coulomb resummation performed here and in $[12,16]$, whereas a large NNLL correction is observed relative to the $\mathrm{NLL}_{s}$ prediction that does not include Coulomb resummation. This observation is consistent with the results of Mellin-space resummation [20]. The effect of Coulomb resummation beyond NNLO and the bound-state effects can be seen by comparing the NNLL and NNLL $_{\text {fixed-C }}$ results and is important in particular for squark-antisquark and gluino-pair production. For squark-squark production, the effect of Coulomb resummation beyond 


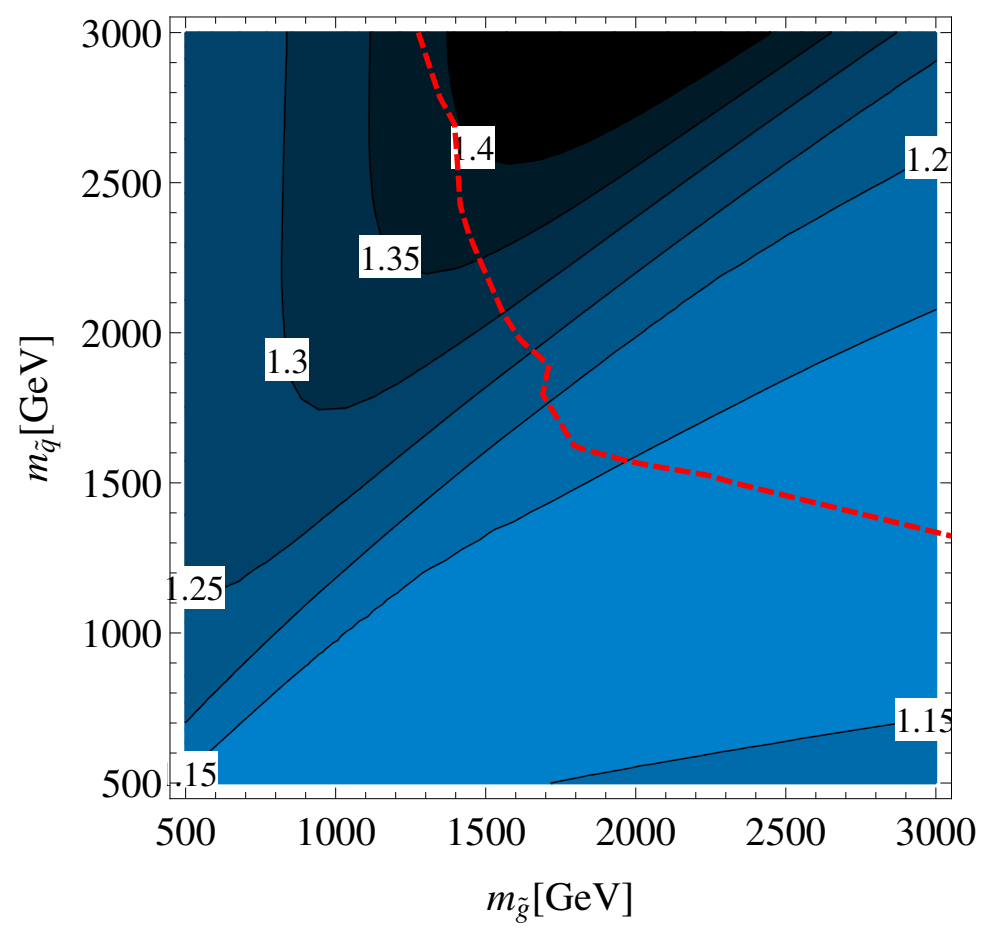

Figure 4. NNLL $K$-factor for the total SUSY pair production rate at the LHC with $\sqrt{s}=13 \mathrm{TeV}$, as a function of the squark mass $m_{\tilde{q}}$ and gluino mass $m_{\tilde{g}}$. The red, dashed line is the ATLAS exclusion bound from searches at $\sqrt{s}=8 \mathrm{TeV}$ in a simplified model with a massless neutralino [2].

NNLO is small. A similar behaviour was also observed for the NLL corrections beyond NLO in [16] and originates from cancellations between the negative corrections arising from the repulsive colour-sextet channel and the positive corrections arising from the attractive colour-triplet channel. The comparison to the approximate NNLO results shows that corrections beyond NNLO become sizeable beyond sparticle masses of about $1.5 \mathrm{TeV}$. The NNLL $_{\text {fixed-C }}$ correction factors in figure 3 cannot be directly compared to the corresponding results using the Mellin-space formalism [20] that are given using the MSTW2008 set of PDFs. However, a comparison to our own earlier results for the NNLL $\mathrm{f}_{\text {fixed-C }}$ approximation at $8 \mathrm{TeV}$ using the same PDFs [19] shows overall good agreement, which is reassuring given the different methods used for the resummation. As already noted in [20], the largest difference of order $10 \%$ appears for gluino pair-production at large masses, which is larger than the estimated resummation uncertainty of approximately $5 \%$ of our result. Therefore, a more detailed comparison of the two approaches will be useful.

Figure 4 shows the NNLL $K$-factor for the total SUSY production rate, i.e. the sum of all squark and gluino pair production processes, at the $13 \mathrm{TeV}$ LHC as a contour plot in the $\left(m_{\tilde{g}}, m_{\tilde{q}}\right)$-plane. The NNLL corrections are larger in the region with $m_{\tilde{g}}<m_{\tilde{q}}$ where squark-gluino and gluino-pair production with the corresponding larger $K$-factors dominate the total SUSY production rate, see e.g. [16]. In contrast, for $m_{\tilde{q}} \leq m_{\tilde{g}}$ the total rate is dominated by squark-squark production with a resulting smaller $K$-factor. Since squark-antisquark production is suppressed compared to squark-squark production 

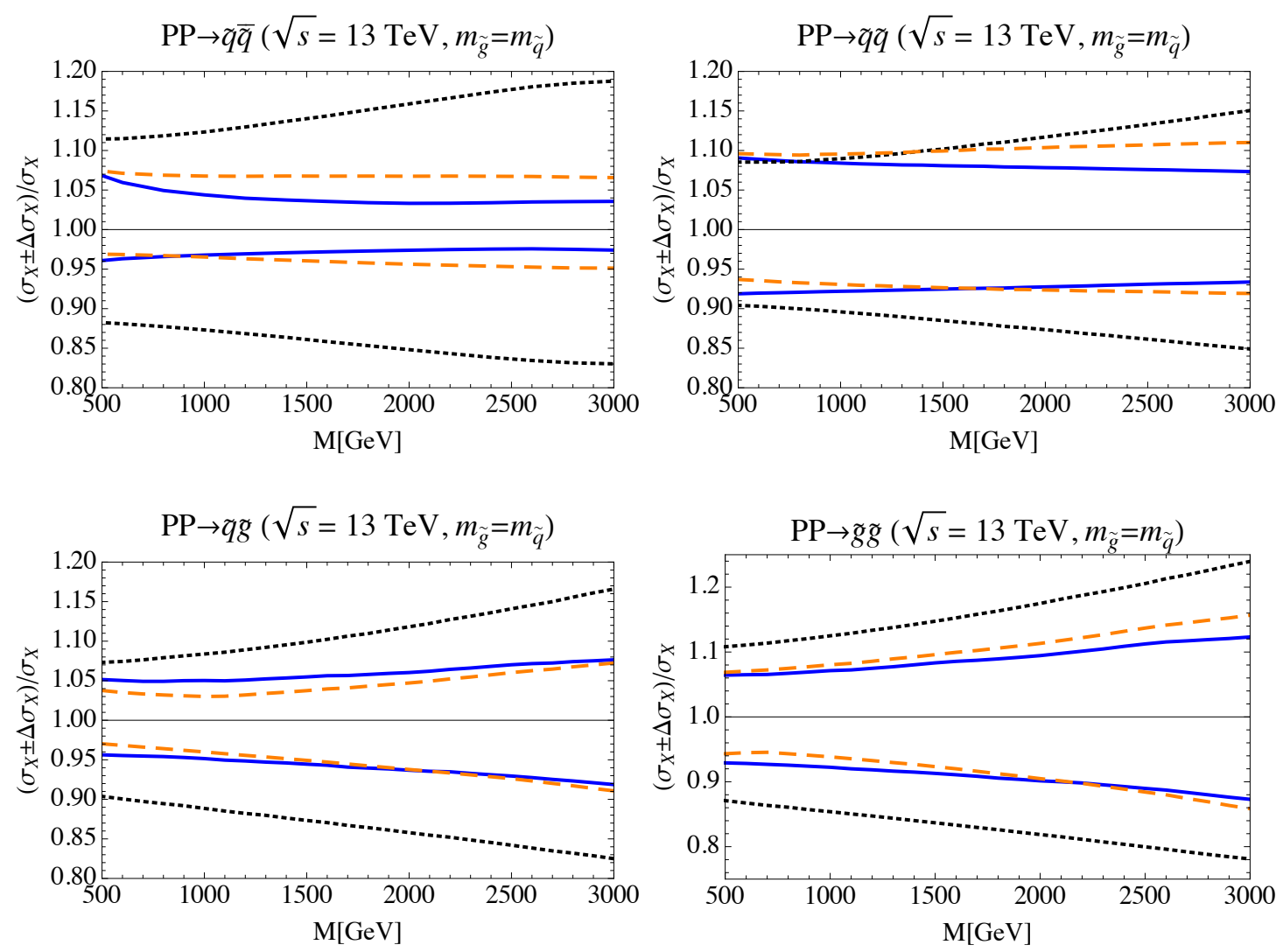

Figure 5. Total theoretical uncertainty (excluding PDF uncertainty) of the NLO approximation (dotted black), NLL (dashed orange) and NNLL (solid blue) resummed results at the LHC with $\sqrt{s}=13 \mathrm{TeV}$. The uncertainty estimate is given by the scale uncertainty, the resummation uncertainty (for NLL and NNLL) and the estimate of missing higher-order corrections (for NNLL). All cross sections are normalized to the one at the central value of the scales.

at the LHC unless $m_{\tilde{g}} \gg m_{\tilde{q}}$, the large $K$-factor in this process has little impact on the total SUSY production rate for the mass range considered in figure 4. For illustration, the plot also shows a Run 1 ATLAS exclusion bound in a simplified model with a massless neutralino [2] that shows that corrections larger than $40 \%$ arise in a region relevant for current searches.

The estimate of the theory uncertainty of the NLO, NLL and NNLL approximations is shown in figure 5, normalized to the central value of the respective prediction. Following the discussion in section 3.1, the NLO uncertainty is estimated by the factorization scale variation, the NLL uncertainty includes in addition the estimate of the resummation ambiguities and the NNLL uncertainty further includes the estimate of the two-loop constant. The $\mathrm{PDF}+\alpha_{s}$ uncertainty is not included in the results shown in figure 5. One observes that the uncertainty is reduced from up to $20 \%$ at NLO to the $10 \%$ level at NLL. While a significant reduction of the uncertainty compared to the NLL results is observed for the squark-antisquark production process at NNLL, only a slight reduction or even an increase is observed for the other processes. We further investigate the different sources of uncer- 
tainties in figure 6 and table 7 below, where it is seen that this behaviour is due to the scale uncertainty, while the resummation uncertainty is generally reduced at NNLL.

Numerical predictions for the cross sections of squark and gluino production at the LHC at $\sqrt{s}=13 \mathrm{TeV}$ are presented in table 5 for a sample of squark and gluino masses from $1-3 \mathrm{TeV}$. The corresponding results for $\sqrt{s}=14 \mathrm{TeV}$ are shown in table 6 for the mass range from $1.5-3.5 \mathrm{TeV}$. In these results, we included the theoretical uncertainty (scale, resummation and higher-order uncertainty as in figure 5) and the $\mathrm{PDF}+\alpha_{s}$ uncertainty determined as discussed in section 3.1. For the processes involving squarks, the PDF $+\alpha_{s}$ uncertainty is of the order of $\pm 5-10 \%$ for lighter sparticle masses. For heavier sparticles, the larger uncertainty in the gluon PDF becomes visible, which leads to a growth of the $\mathrm{PDF}+\alpha_{s}$ uncertainty to $\pm 30 \%$ for squark-gluino and over $\pm 100 \%$ for squark-antisquark production at $\sqrt{s}=14 \mathrm{TeV}$. For the gluino-pair production process the PDF uncertainty grows from $\pm 20 \%$ to over $\pm 80 \%$ at the highest considered masses at $\sqrt{s}=14 \mathrm{TeV}$. For the squark-squark production process, where the gluon PDF does not enter at tree level, the relative $\mathrm{PDF}+\alpha_{s}$ uncertainty is smaller and remains below $\pm 10 \%$ throughout the mass range. Therefore the PDF uncertainty is smaller than the NLO scale uncertainty or comparable at smaller masses, whereas the uncertainty due to the poorly determined gluon PDF becomes very large at high masses, in particular for squark-antisquark and gluino-pair production. It should be taken into account, however, that the largest PDF uncertainties appear for cross sections of the order of $10^{-7} \mathrm{pb}$ that are beyond the reach of even the high-luminosity phase of the LHC. In general, the NLL resummation reduces the theory uncertainty below the PDF uncertainty, apart from squark-squark production where the PDF uncertainties are very small and typically below the theory uncertainties. Consistent with figure 5, the NNLL resummation further reduces the theory uncertainty strongly for squark-antisquark production, whereas the effect for the other processes is moderate. The size of the NNLL corrections is consistent with figure 3, with corrections relative to NLO of up to a factor of two for gluino pair production at the highest considered masses. Although the PDF4LHC15 set combines the results of several PDF fits, it should be taken into account, however, that different PDF sets can lead to results that are not covered by the PDF4LHC15 error estimate, or have a much larger estimate of the PDF uncertainty, in particular for processes involving the gluon PDF [17, 75, 80]. We refer to [81] for a recent discussion of the effect of different sets of PDF fits on predictions with NLL soft-gluon resummation. This includes a PDF set obtained using threshold-resummed cross sections in the PDF fit [82] that is in principle appropriate for resummed calculations, but currently has large uncertainties due to a reduced data set used in the fit. For squark and gluino production, non-trivial changes on the central values were found for the resummed PDFs that, however, lie inside of the uncertainty band of the standard PDF sets.

The scale dependence of various higher-order approximations is shown in figure 6 . In addition to the approximate NNLO corrections, the full NNLL result and the NNLL soft resummation with fixed-order Coulomb corrections NNLL $_{\text {fixed-C, we also consider an }}$ approximation $\mathrm{NNLL}_{\mathrm{sh}}$ where Coulomb corrections are set to zero in the resummation formula and only included through the matching to $\mathrm{NNLO}_{\text {app }}$. It is seen that soft resummation in $\mathrm{NNLL}_{\mathrm{sh}}$ significantly affects the shape of the scale dependence and reduces the scale uncertainty for squark-antisquark and squark-squark production. For the mass values considered here, the approximate NNLO cross section has a maximum near the default 


\begin{tabular}{|c|c|c|c|c|c|}
\hline$m_{\tilde{q}}, m_{\tilde{g}}(\mathrm{TeV})$ & $\tilde{s} \tilde{s}^{\prime}$ & $\sigma_{\mathrm{NLO}}(\mathrm{pb})$ & $\sigma_{\mathrm{NLL}}(\mathrm{pb})$ & $\sigma_{\mathrm{NNLL}}(\mathrm{pb})$ & $K_{\mathrm{NNLL}}$ \\
\hline \multirow[t]{4}{*}{$1.3,1.5$} & $\tilde{q} \overline{\tilde{q}}$ & $2.81_{-0.37_{-0.18}^{+0.36+0.18} \times 10^{-2}}^{-2}$ & $3.06_{-0.11-0.19}^{+0.19+0.19} \times 10^{-2}$ & $3.49_{-0.09-0.20}^{+0.13+0.20} \times 10^{-2}$ & 1.24 \\
\hline & $\tilde{q} \tilde{q}$ & $8.64_{-0.90-0.25}^{+0.74+0.25} \times 10^{-2}$ & $8.76_{-0.55-0.25}^{+0.76+0.25} \times 10^{-2}$ & $9.37_{-0.53-0.26}^{+0.56+0.26} \times 10^{-2}$ & 1.08 \\
\hline & $\tilde{q} \tilde{g}$ & $7.25_{-0.86-0.50}^{+0.62+0.50} \times 10^{-2}$ & $7.93_{-0.37-0.51}^{+0.28+0.51} \times 10^{-2}$ & $8.49_{-0.46-0.52}^{+0.43+0.52} \times 10^{-2}$ & 1.17 \\
\hline & $\tilde{g} \tilde{g}$ & $6.39_{-1.01-1.34}^{+0.88+1.34} \times 10^{-3}$ & $8.03_{-0.66-1.37}^{+0.81+1.37} \times 10^{-3}$ & $8.71_{-0.89-1.40}^{+0.82+1.40} \times 10^{-3}$ & 1.36 \\
\hline \multirow[t]{4}{*}{$1.5,1.3$} & $\tilde{\tilde{q}}$ & $9.33_{-1.26-0.70}^{+1.23+0.70} \times 10^{-3}$ & $1.02_{-0.04-0.07}^{+0.06+0.07} \times 10^{-2}$ & $1.17_{-0.04-0.08}^{+0.05+0.08} \times 10^{-2}$ & 1.25 \\
\hline & $\tilde{q} \tilde{q}$ & $4.20_{-0.50-0.13}^{+0.47+0.13} \times 10^{-2}$ & $4.25_{-0.35-0.13}^{+0.46+0.13} \times 10^{-2}$ & $4.58_{-0.44-0.14}^{+0.47+0.14} \times 10^{-2}$ & 1.09 \\
\hline & $\tilde{q} \tilde{g}$ & $8.70_{-1.15-0.60}^{+0.970 .60} \times 10^{-2}$ & $9.59_{-0.47-0.61}^{+0.35+0.61} \times 10^{-2}$ & $1.06_{-0.05-0.06}^{+0.06+0.06} \times 10^{-1}$ & 1.22 \\
\hline & $\tilde{g} \tilde{g}$ & $2.45_{-0.40-0.42}^{+0.38+0.42} \times 10^{-2}$ & $2.97_{-0.19-0.43}^{+0.24+0.43} \times 10^{-2}$ & $3.36_{-0.22-0.45}^{+0.22+0.45} \times 10^{-2}$ & 1.37 \\
\hline \multirow[t]{4}{*}{$1.8,2$} & $\tilde{\tilde{q}}$ & $1.66_{-0.24-0.17}^{+0.24+0.17} \times 10^{-3}$ & $1.87_{-0.08-0.18}^{+0.12+0.18} \times 10^{-3}$ & $2.16_{-0.05-0.19}^{+0.07+0.19} \times 10^{-3}$ & 1.30 \\
\hline & $\tilde{q} \tilde{q}$ & $9.61_{-1.13-0.34}^{+0.99+0.34} \times 10^{-3}$ & $9.82_{-0.68-0.34}^{+0.92+0.34} \times 10^{-3}$ & $1.06_{-0.06-0.04}^{+0.07+0.04} \times 10^{-2}$ & 1.11 \\
\hline & $\tilde{q} \tilde{g}$ & $5.15_{-0.69-0.59}^{+0.54+0.59} \times 10^{-3}$ & $5.86_{-0.35-0.60}^{+0.27+0.60} \times 10^{-3}$ & $6.34_{-0.39-0.60}^{+0.36+0.60} \times 10^{-3}$ & 1.23 \\
\hline & $\tilde{g} \tilde{g}$ & $3.18_{-0.56-1.05}^{+0.51+1.05} \times 10^{-4}$ & $4.33_{-0.45-1.08}^{+0.52+1.08} \times 10^{-4}$ & $4.71_{-0.54-1.12}^{+0.50+1.12} \times 10^{-4}$ & 1.48 \\
\hline \multirow[t]{4}{*}{$2,1.8$} & $\tilde{\tilde{q}}$ & $5.84_{-0.87-0.79}^{+0.88+0.79} \times 10^{-4}$ & $6.62_{-0.28-0.80}^{+0.42+0.80} \times 10^{-4}$ & $7.69_{-0.23-0.85}^{+0.27+0.85} \times 10^{-4}$ & 1.32 \\
\hline & $\tilde{q} \tilde{q}$ & $4.68_{-0.61-0.19}^{+0.58+0.19} \times 10^{-3}$ & $4.78_{-0.40-0.19}^{+0.53+0.19} \times 10^{-3}$ & $5.23_{-0.45-0.19}^{+0.49+0.19} \times 10^{-3}$ & 1.12 \\
\hline & $\tilde{q} \tilde{g}$ & $5.96_{-0.86-0.68}^{+0.76+0.68} \times 10^{-3}$ & $6.82_{-0.41-0.69}^{+0.28+0.69} \times 10^{-3}$ & $7.62_{-0.44-0.70}^{+0.46+0.70} \times 10^{-3}$ & 1.28 \\
\hline & $\tilde{g} \tilde{g}$ & $1.07_{-0.19-0.30}^{+0.19+0.30} \times 10^{-3}$ & $1.40_{-0.11-0.30}^{+0.14+0.30} \times 10^{-3}$ & $1.60_{-0.13-0.32}^{+0.13+0.32} \times 10^{-3}$ & 1.49 \\
\hline \multirow[t]{4}{*}{$2.3,2.5$} & $\tilde{q} \overline{\tilde{q}}$ & $1.13_{-0.18-0.24}^{+0.19+0.24} \times 10^{-4}$ & $1.32_{-0.06-0.25}^{+0.09+0.25} \times 10^{-4}$ & $1.55_{-0.04-0.26}^{+0.05+0.26} \times 10^{-4}$ & 1.37 \\
\hline & $\tilde{q} \tilde{q}$ & $1.18_{-0.15-0.06}^{+0.14+0.06} \times 10^{-3}$ & $1.21_{-0.09-0.06}^{+0.12+0.06} \times 10^{-3}$ & $1.33_{-0.08-0.06}^{+0.09+0.06} \times 10^{-3}$ & 1.13 \\
\hline & $\tilde{q} \tilde{g}$ & $4.29_{-0.65-0.77}^{+0.55+0.77} \times 10^{-4}$ & $5.13_{-0.37-0.78}^{+0.30+0.78} \times 10^{-4}$ & $5.60_{-0.39-0.79}^{+0.37+0.79} \times 10^{-4}$ & 1.31 \\
\hline & $\tilde{g} \tilde{g}$ & $1.88_{-0.36-0.93}^{+0.36+0.93} \times 10^{-5}$ & $2.84_{-0.36-0.98}^{+0.41+0.98} \times 10^{-5}$ & $3.11_{-0.39-1.04}^{+0.38+1.04} \times 10^{-5}$ & 1.65 \\
\hline \multirow[t]{4}{*}{$2.5,2.3$} & $\tilde{q} \overline{\tilde{q}}$ & $3.98_{-0.64-1.27}^{+0.68+1.27} \times 10^{-5}$ & $4.72_{-0.22-1.29}^{+0.30+1.29} \times 10^{-5}$ & $5.56_{-0.15-1.36}^{+0.20+1.36} \times 10^{-5}$ & 1.40 \\
\hline & $\tilde{q} \tilde{q}$ & $5.55_{-0.79-0.33}^{+0.77+0.33} \times 10^{-4}$ & $5.74_{-0.48}^{+0.65+0.33} \times 10^{-4}$ & $6.36_{-0.51-0.33}^{+0.56+0.33} \times 10^{-4}$ & 1.15 \\
\hline & $\tilde{q} \tilde{g}$ & $4.84_{-0.77-0.86}^{+0.71+0.86} \times 10^{-4}$ & $5.83_{-0.41-0.88}^{+0.31+0.88} \times 10^{-4}$ & $6.56_{-0.43-0.90}^{+0.45+0.90} \times 10^{-4}$ & 1.36 \\
\hline & $\tilde{g} \tilde{g}$ & $6.07_{-1.20-2.56}^{+1.25+2.56} \times 10^{-5}$ & $8.62_{-0.85-2.66}^{+1.03+2.66} \times 10^{-5}$ & $1.00_{-0.09-0.28}^{+0.10+0.28} \times 10^{-4}$ & 1.65 \\
\hline \multirow[t]{4}{*}{$2.8,3$} & $\tilde{\tilde{q}}$ & $7.94_{-1.33-4.56}^{+1.45+4.56} \times 10^{-6}$ & $9.69_{-0.48-4.65}^{+0.63+4.65} \times 10^{-6}$ & $1.15_{-0.03-0.48}^{+0.04+0.48} \times 10^{-5}$ & 1.45 \\
\hline & $\tilde{q} \tilde{q}$ & $1.41_{-0.20-0.11}^{+0.19+0.11} \times 10^{-4}$ & $1.47_{-0.11-0.11}^{+0.15+0.11} \times 10^{-4}$ & $1.64_{-0.10-0.11}^{+0.11+0.11} \times 10^{-4}$ & 1.17 \\
\hline & $\tilde{q} \tilde{g}$ & $3.62_{-0.61-0.97}^{+0.56+0.97} \times 10^{-5}$ & $4.62_{-0.40-0.99}^{+0.32+0.99} \times 10^{-5}$ & $5.10_{-0.41-1.01}^{+0.37+1.01} \times 10^{-5}$ & 1.41 \\
\hline & $\tilde{g} \tilde{g}$ & $1.15_{-0.24-0.82}^{+0.25+0.82} \times 10^{-6}$ & $1.97_{-0.31-0.92}^{+0.33+0.92} \times 10^{-6}$ & $2.18_{-0.31-1.01}^{+0.29+1.01} \times 10^{-6}$ & 1.90 \\
\hline \multirow[t]{4}{*}{$3,2.8$} & $\overline{\tilde{q}}$ & $2.83_{-0.47-2.53}^{+0.51+2.53} \times 10^{-6}$ & $3.50_{-0.17-2.56}^{+0.22+2.56} \times 10^{-6}$ & $4.17_{-0.12-2.63}^{+0.15+2.63} \times 10^{-6}$ & 1.47 \\
\hline & $\tilde{q} \tilde{q}$ & $6.28_{-0.96-0.60}^{+0.98+0.60} \times 10^{-5}$ & $6.61_{-0.56-0.59}^{+0.77+0.59} \times 10^{-5}$ & $7.43_{-0.56-0.59}^{+0.61+0.59} \times 10^{-5}$ & 1.18 \\
\hline & $\tilde{q} \tilde{g}$ & $4.02_{-0.71-1.08}^{+0.69+1.08} \times 10^{-5}$ & $5.16_{-0.43-1.10}^{+0.34+1.10} \times 10^{-5}$ & $5.87_{-0.44-1.13}^{+0.44+1.13} \times 10^{-5}$ & 1.46 \\
\hline & $\tilde{g} \tilde{g}$ & $3.70_{-0.80-2.27}^{+0.88+2.27} \times 10^{-6}$ & $5.90_{-0.73-2.45}^{+0.85+2.45} \times 10^{-6}$ & $6.94_{-0.76-2.70}^{+0.78+2.70} \times 10^{-6}$ & 1.87 \\
\hline
\end{tabular}

Table 5. Predictions for the LHC with $\sqrt{s}=13 \mathrm{TeV}$ using the PDF4LHC15_nnlo_30 PDFs. The first and second error refer to the theoretical uncertainty, defined as in figure 5 , and the PDF $+\alpha_{s}$ uncertainty, respectively. 


\begin{tabular}{|c|c|c|c|c|c|}
\hline$m_{\tilde{q}}, m_{\tilde{g}}(\mathrm{TeV})$ & $\tilde{s} \tilde{s}^{\prime}$ & $\sigma_{\mathrm{NLO}}(\mathrm{pb})$ & $\sigma_{\mathrm{NLL}}(\mathrm{pb})$ & $\sigma_{\mathrm{NNLL}}(\mathrm{pb})$ & $K_{\mathrm{NNLL}}$ \\
\hline \multirow[t]{4}{*}{$1.8,2$} & $\tilde{q} \overline{\tilde{q}}$ & $2.82_{-0.40-0.26}^{+0.40+0.26} \times 10^{-3}$ & $3.14_{-0.13-0.26}^{+0.20+0.26} \times 10^{-3}$ & $3.61_{-0.09-0.28}^{+0.12+0.28} \times 10^{-3}$ & 1.28 \\
\hline & $\tilde{q} \tilde{q}$ & $1.40_{-0.16-0.05}^{+0.14+0.05} \times 10^{-2}$ & $1.43_{-0.10-0.05}^{+0.13+0.05} \times 10^{-2}$ & $1.54_{-0.09-0.05}^{+0.10+0.05} \times 10^{-2}$ & 1.10 \\
\hline & $\tilde{q} \tilde{g}$ & $8.61_{-1.11-0.86}^{+0.85+0.86} \times 10^{-3}$ & $9.68_{-0.53-0.87}^{+0.41+0.87} \times 10^{-3}$ & $1.04_{-0.06-0.09}^{+0.06+0.09} \times 10^{-2}$ & 1.21 \\
\hline & $\tilde{g} \tilde{g}$ & $6.02_{-1.01-1.75}^{+0.91+1.75} \times 10^{-4}$ & $7.97_{-0.78-1.80}^{+0.91+1.80} \times 10^{-4}$ & $8.63_{-0.96-1.84}^{+0.89+1.84} \times 10^{-4}$ & 1.44 \\
\hline \multirow[t]{4}{*}{$2 ., 1.8$} & $\tilde{q} \overline{\tilde{q}}$ & $1.06_{-0.15-0.12}^{+0.15+0.12} \times 10^{-3}$ & $1.19_{-0.05-0.12}^{+0.07+0.12} \times 10^{-3}$ & $1.37_{-0.04-0.13}^{+0.05+0.13} \times 10^{-3}$ & 1.29 \\
\hline & $\tilde{q} \tilde{q}$ & $7.20_{-0.90-0.26}^{+0.85+0.26} \times 10^{-3}$ & $7.34_{-0.59-0.26}^{+0.80+0.26} \times 10^{-3}$ & $7.98_{-0.70-0.27}^{+0.75+0.27} \times 10^{-3}$ & 1.11 \\
\hline & $\tilde{q} \tilde{g}$ & $9.94_{-1.39-0.99}^{+1.20+0.99} \times 10^{-3}$ & $1.12_{-0.06-0.10}^{+0.04+0.10} \times 10^{-2}$ & $1.25_{-0.07-0.10}^{+0.07+0.10} \times 10^{-2}$ & 1.26 \\
\hline & $\tilde{g} \tilde{g}$ & $1.91_{-0.33-0.47}^{+0.32+0.47} \times 10^{-3}$ & $2.43_{-0.18-0.48}^{+0.22+0.48} \times 10^{-3}$ & $2.77_{-0.21-0.50}^{+0.21+0.50} \times 10^{-3}$ & 1.45 \\
\hline \multirow[t]{4}{*}{$2.3,2.5$} & $\tilde{\tilde{q}}$ & $2.28_{-0.35-0.38}^{+0.36+0.38} \times 10^{-4}$ & $2.62_{-0.12-0.38}^{+0.17+0.38} \times 10^{-4}$ & $3.05_{-0.07-0.41}^{+0.10+0.41} \times 10^{-4}$ & 1.34 \\
\hline & $\tilde{q} \tilde{q}$ & $1.96_{-0.25-0.09}^{+0.22+0.09} \times 10^{-3}$ & $2.02_{-0.14-0.09}^{+0.20+0.09} \times 10^{-3}$ & $2.20_{-0.13-0.09}^{+0.14+0.09} \times 10^{-3}$ & 1.12 \\
\hline & $\tilde{q} \tilde{g}$ & $8.31_{-1.20-1.27}^{+0.99+1.27} \times 10^{-4}$ & $9.75_{-0.65-1.29}^{+0.52+1.29} \times 10^{-4}$ & $1.06_{-0.07-0.13}^{+0.07+0.13} \times 10^{-3}$ & 1.28 \\
\hline & $\tilde{g} \tilde{g}$ & $4.21_{-0.78-1.81}^{+0.74+1.81} \times 10^{-5}$ & $6.08_{-0.72-1.88}^{+0.83+1.88} \times 10^{-5}$ & $6.64_{-0.79-1.97}^{+0.77+1.97} \times 10^{-5}$ & 1.58 \\
\hline \multirow[t]{4}{*}{$2.5,2.3$} & $\tilde{q} \bar{q}$ & $8.62_{-1.34-1.96}^{+1.40+1.96} \times 10^{-5}$ & $1.00_{-0.04-0.20}^{+0.06+0.20} \times 10^{-4}$ & $1.18_{-0.03-0.21}^{+0.04+0.21} \times 10^{-4}$ & 1.36 \\
\hline & $\tilde{q} \tilde{q}$ & $9.89_{-1.34-0.50}^{+1.30+0.50} \times 10^{-4}$ & $1.02_{-0.08-0.05}^{+0.11+0.05} \times 10^{-3}$ & $1.12_{-0.09-0.05}^{+0.10+0.05} \times 10^{-3}$ & 1.13 \\
\hline & $\tilde{q} \tilde{g}$ & $9.38_{-1.43-1.43}^{+1.29+1.43} \times 10^{-4}$ & $1.11_{-0.07-0.15}^{+0.05+0.15} \times 10^{-3}$ & $1.24_{-0.08-0.15}^{+0.08+0.15} \times 10^{-3}$ & 1.32 \\
\hline & $\tilde{g} \tilde{g}$ & $1.27_{-0.24-0.46}^{+0.24+0.46} \times 10^{-4}$ & $1.74_{-0.16-0.48}^{+0.19+0.48} \times 10^{-4}$ & $2.00_{-0.17-0.50}^{+0.18+0.50} \times 10^{-4}$ & 1.58 \\
\hline \multirow[t]{4}{*}{$2.8,3$} & $\tilde{q} \overline{\tilde{q}}$ & $1.91_{-0.31-0.73}^{+0.34+0.73} \times 10^{-5}$ & $2.29_{-0.11-0.74}^{+0.15+0.74} \times 10^{-5}$ & $2.70_{-0.06-0.78}^{+0.09+0.78} \times 10^{-5}$ & 1.41 \\
\hline & $\tilde{q} \tilde{q}$ & $2.77_{-0.38-0.18}^{+0.36+0.18} \times 10^{-4}$ & $2.88_{-0.22-0.18}^{+0.29+0.18} \times 10^{-4}$ & $3.19_{-0.19-0.18}^{+0.21+0.18} \times 10^{-4}$ & 1.15 \\
\hline & $\tilde{q} \tilde{g}$ & $8.41_{-1.35-1.91}^{+1.19+1.91} \times 10^{-5}$ & $1.04_{-0.08-0.19}^{+0.07+0.19} \times 10^{-4}$ & $1.14_{-0.09-0.20}^{+0.08+0.20} \times 10^{-4}$ & 1.36 \\
\hline & $\tilde{g} \tilde{g}$ & $3.13_{-0.63-1.91}^{+0.64+1.91} \times 10^{-6}$ & $5.05_{-0.72-2.07}^{+0.79+2.07} \times 10^{-6}$ & $5.56_{-0.75-2.23}^{+0.70+2.23} \times 10^{-6}$ & 1.78 \\
\hline \multirow[t]{4}{*}{$3 ., 2.8$} & $\tilde{\tilde{q}}$ & $7.24_{-1.18-4.15}^{+1.27+4.15} \times 10^{-6}$ & $8.78_{-0.42-4.22}^{+0.55+4.22} \times 10^{-6}$ & $1.04_{-0.03-0.44}^{+0.04+0.44} \times 10^{-5}$ & 1.44 \\
\hline & $\tilde{q} \tilde{q}$ & $1.34_{-0.20-0.10}^{+0.20+0.10} \times 10^{-4}$ & $1.40_{-0.12-0.10}^{+0.16+0.10} \times 10^{-4}$ & $1.56_{-0.12-0.10}^{+0.13+0.10} \times 10^{-4}$ & 1.16 \\
\hline & $\tilde{q} \tilde{g}$ & $9.34_{-1.56-2.12}^{+1.48+2.12} \times 10^{-5}$ & $1.16_{-0.09-0.21}^{+0.07+0.21} \times 10^{-4}$ & $1.31_{-0.10-0.22}^{+0.09+0.22} \times 10^{-4}$ & 1.41 \\
\hline & $\tilde{g} \tilde{g}$ & $9.30_{-1.92-4.90}^{+2.05+4.90} \times 10^{-6}$ & $1.40_{-0.16-0.52}^{+0.19+0.52} \times 10^{-5}$ & $1.64_{-0.17-0.56}^{+0.17+0.56} \times 10^{-5}$ & 1.76 \\
\hline \multirow[t]{4}{*}{$3.3,3.5$} & $\tilde{q} \overline{\tilde{q}}$ & $1.68_{-0.28-1.66}^{+0.31+1.66} \times 10^{-6}$ & $2.07_{-0.10-1.68}^{+0.13+1.68} \times 10^{-6}$ & $2.46_{-0.06-1.73}^{+0.08+1.73} \times 10^{-6}$ & 1.47 \\
\hline & $\tilde{q} \tilde{q}$ & $3.68_{-0.55-0.38}^{+0.54+0.38} \times 10^{-5}$ & $3.89_{-0.30-0.37}^{+0.41+0.37} \times 10^{-5}$ & $4.35_{-0.26-0.37}^{+0.28+0.37} \times 10^{-5}$ & 1.18 \\
\hline & $\tilde{q} \tilde{g}$ & $8.18_{-1.44-2.68}^{+1.37+2.68} \times 10^{-6}$ & $1.08_{-0.10-0.27}^{+0.08+0.27} \times 10^{-5}$ & $1.20_{-0.10-0.28}^{+0.09+0.28} \times 10^{-5}$ & 1.47 \\
\hline & $\tilde{g} \tilde{g}$ & $2.28_{-0.50-1.90}^{+0.53+1.90} \times 10^{-7}$ & $4.19_{-0.69-2.26}^{+0.74+2.26} \times 10^{-7}$ & $4.65_{-0.69-2.55}^{+0.66+2.55} \times 10^{-7}$ & 2.04 \\
\hline \multirow[t]{4}{*}{$3.5,3.3$} & $\tilde{q} \overline{\tilde{q}}$ & $6.72_{-1.11-9.60}^{+1.20+9.60} \times 10^{-7}$ & $8.32_{-0.39-9.65}^{+0.52+9.65} \times 10^{-7}$ & $9.93_{-0.28-9.82}^{+0.35+9.82} \times 10^{-7}$ & 1.48 \\
\hline & $\tilde{q} \tilde{q}$ & $1.68_{-0.27-0.21}^{+0.27+0.21} \times 10^{-5}$ & $1.78_{-0.15-0.20}^{+0.21+0.20} \times 10^{-5}$ & $2.02_{-0.14-0.20}^{+0.16+0.20} \times 10^{-5}$ & 1.20 \\
\hline & $\tilde{q} \tilde{g}$ & $8.98_{-1.63-2.93}^{+1.63+2.93} \times 10^{-6}$ & $1.19_{-0.11-0.30}^{+0.09+0.30} \times 10^{-5}$ & $1.36_{-0.11-0.31}^{+0.11+0.31} \times 10^{-5}$ & 1.52 \\
\hline & $\tilde{g} \tilde{g}$ & $6.89_{-1.53-5.02}^{+1.72+5.02} \times 10^{-7}$ & $1.17_{-0.16-0.57}^{+0.18+0.57} \times 10^{-6}$ & $1.38_{-0.16-0.64}^{+0.16+0.64} \times 10^{-6}$ & 2.01 \\
\hline
\end{tabular}

Table 6. Predictions for the LHC with $\sqrt{s}=14 \mathrm{TeV}$. The errors are defined as in table 5 . 

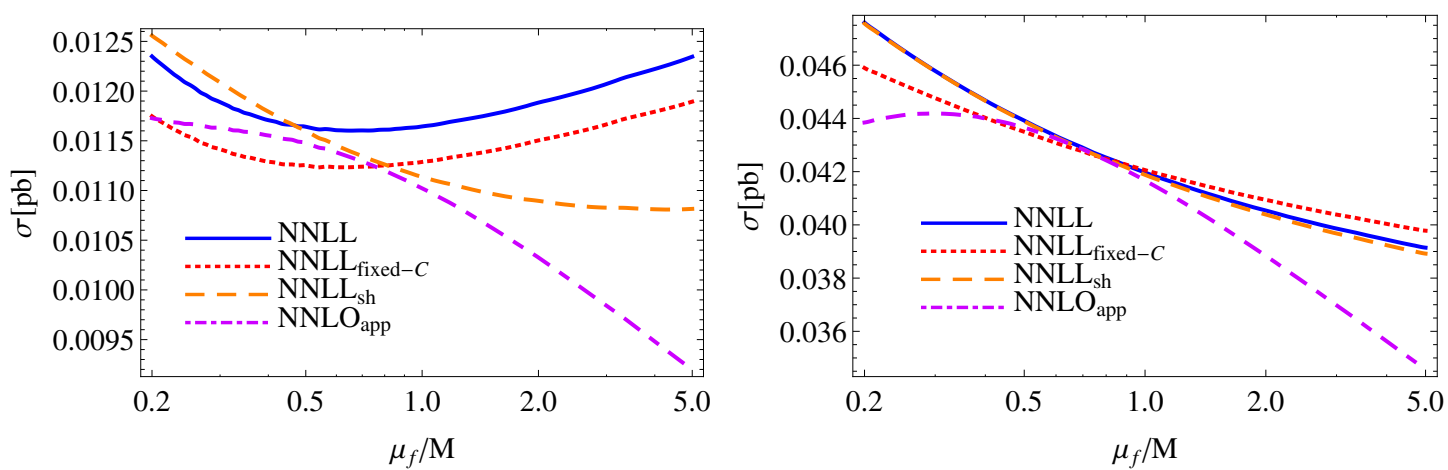

$\mathrm{PP} \rightarrow \tilde{q} \tilde{g}\left(\sqrt{s}=13 \mathrm{TeV}, m_{\tilde{g}}=m_{\tilde{q}}=1.5 \mathrm{TeV}\right)$

$$
\mathrm{PP} \rightarrow \tilde{g} \tilde{g}\left(\sqrt{s}=13 \mathrm{TeV}, m_{\tilde{g}}=m_{\tilde{q}}=1.5 \mathrm{TeV}\right)
$$
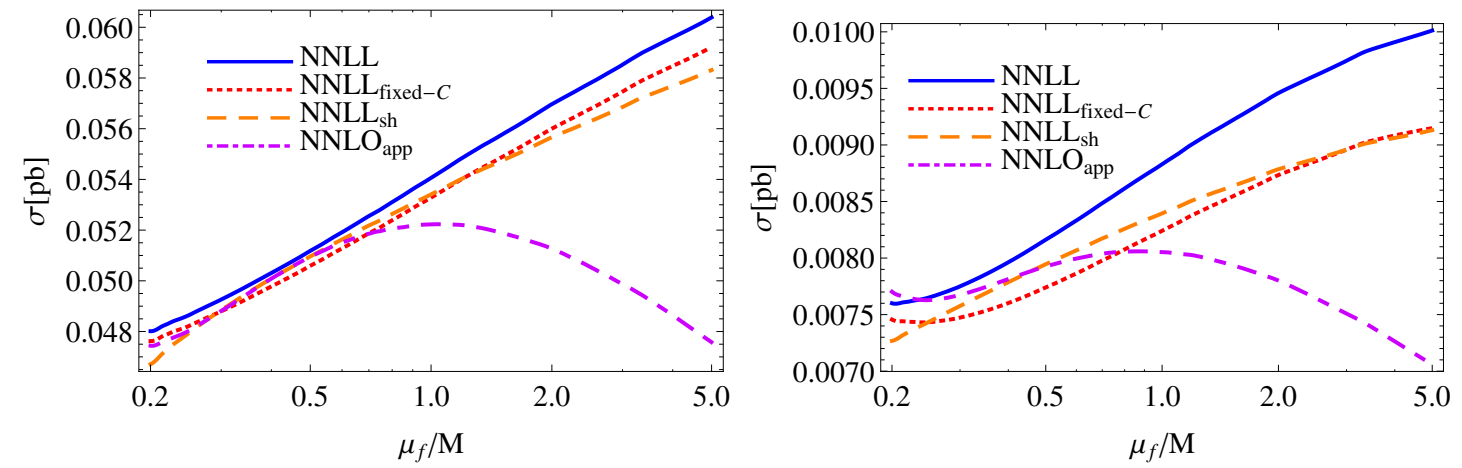

Figure 6. Cross section for squark and gluino production at the LHC with $\sqrt{s}=13 \mathrm{TeV}$ for full NNLL resummation (solid blue), NNLL with fixed-order Coulomb corrections (dotted red), $\mathrm{NNLL}_{\text {sh }}$ (dashed orange) and approximate NNLO (dot-dashed pink), as a function of the ratio of the factorization scale and average produced mass.

scale $\mu_{f}=M$, so the variation of the factorization scale alone leads to an asymmetrical error estimate, whereas the soft resummation leads to a more symmetrical behaviour. The inclusion of soft-Coulomb interference in the NNLL $\mathrm{fixed}_{\mathrm{C}}$ prediction further reduces the uncertainty for the case of squark-antisquark and squark-squark production, while it is increased for gluino-pair and squark-gluino production. This observation is consistent with the results obtained in the Mellin-space approach for gluino-pair production [20]. The further resummation of Coulomb corrections in the NNLL prediction provides an overall shift of the cross section while the scale dependence is qualitatively similar to the $\mathrm{NNLL}_{\text {fixed-C }}$ approximation.

In table 7 we provide numerical results for the higher-order approximations NLL, $\mathrm{NLLO}_{\text {app }}$, NNLL fixed-C and NNLL defined above. In order to study the contributions of the different sources of uncertainties, the scale uncertainty is shown separately from the remaining theoretical uncertainties. The results are shown for the same mass values as in table 5. The magnitude of the corrections from the successive improvement in accuracy is consistent with that seen in figure 3. The difference between the approximate NNLO results and the resummed predictions is moderate at smaller masses but grows more sizeable 


\begin{tabular}{|c|c|c|c|c|c|}
\hline$m_{\tilde{q}}, m_{\tilde{g}}(\mathrm{TeV}$ & s & $\sigma_{\mathrm{NLL}}(\mathrm{pb})$ & $\sigma_{\mathrm{NNLO}_{\text {app }}}(\mathrm{pb})$ & $\sigma_{\mathrm{NNLL}_{\text {fixed-C }}}(\mathrm{pb})$ & $\sigma_{\mathrm{NNLL}}(\mathrm{pb})$ \\
\hline \multirow[t]{4}{*}{$1.3,1.5$} & $\tilde{q} \overline{\tilde{q}}$ & $3.06_{-0.02-0.11}^{+0.14+0.12} \times 10^{-2}$ & $3.32_{-0.18-0.07}^{+0.09+0.07} \times 10^{-2}$ & $3.39_{-0.04-0.09}^{+0.08+0.12} \times 10^{-2}$ & $3.49_{-0.03-0.08}^{+0.09+0.09} \times 10^{-2}$ \\
\hline & $\tilde{q} \tilde{q}$ & $8.76_{-0.55-0.07}^{+0.75+0.09} \times 10^{-2}$ & $9.31_{-0.53-0.46}^{+0.31+0.46} \times 10^{-2}$ & $9.38_{-0.19-0.46}^{+0.21+0.48} \times 10^{-2}$ & $9.37_{-0.25-0.47}^{+0.32+0.46} \times 10^{-2}$ \\
\hline & $\tilde{q} \tilde{g}$ & $7.93_{-0.02-0.37}^{+0.20+0.21} \times 10^{-2}$ & $8.27_{-0.26-0.06}^{+0.00+0.06} \times 10^{-2}$ & $8.38_{-0.42-0.09}^{+0.39+0.07} \times 10^{-2}$ & $8.49_{-0.46-0.08}^{+0.42+0.06} \times 10^{-2}$ \\
\hline & $\tilde{g} \tilde{g}$ & $8.03_{-0.32-0.58}^{+0.55+0.59} \times 10^{-3}$ & $7.97_{-0.25-0.41}^{+0.00+0.41} \times 10^{-3}$ & $8.14_{-0.61-0.42}^{+0.55+0.41} \times 10^{-3}$ & $8.71_{-0.77-0.44}^{+0.69+0.44} \times 10^{-3}$ \\
\hline \multirow[t]{4}{*}{$1.5,1.3$} & $\tilde{q} \tilde{\tilde{q}}$ & $1.02_{-0.01-0.04}^{+0.05+0.04} \times 10^{-2}$ & $1.11_{-0.07-0.04}^{+0.04+0.04} \times 10^{-2}$ & $1.13_{-0.01-0.04}^{+0.02+0.05} \times 10^{-2}$ & $1.17_{-0.01-0.04}^{+0.02+0.04} \times 10^{-2}$ \\
\hline & $\tilde{q} \tilde{q}$ & $4.25_{-0.34-0.04}^{+0.46+0.04} \times 10^{-2}$ & $4.55_{-0.34-0.39}^{+0.26+0.39} \times 10^{-2}$ & $4.59_{-0.15-0.39}^{+0.20+0.40} \times 10^{-2}$ & $4.58_{-0.19-0.39}^{+0.26+0.39} \times 10^{-2}$ \\
\hline & $\tilde{q} \tilde{g}$ & $9.59_{-0.00-0.47}^{+0.23+0.27} \times 10^{-2}$ & $1.02_{-0.03-0.02}^{+0.00+0.02} \times 10^{-1}$ & $1.05_{-0.05-0.03}^{+0.05+0.03} \times 10^{-1}$ & $1.06_{-0.05-0.03}^{+0.06+0.02} \times 10^{-1}$ \\
\hline & $\tilde{g} \tilde{g}$ & $2.97_{-0.03-0.19}^{+0.12+0.20} \times 10^{-2}$ & $3.08_{-0.12-0.08}^{+0.02+0.08} \times 10^{-2}$ & $3.14_{-0.13-0.09}^{+0.15+0.10} \times 10^{-2}$ & $3.36_{-0.19-0.10}^{+0.19+0.10} \times 10^{-2}$ \\
\hline \multirow[t]{4}{*}{$1.8,2$} & $\tilde{q} \overline{\tilde{q}}$ & $1.87_{-0.02-0.08}^{+0.08+0.08} \times 10^{-3}$ & $2.02_{-0.14-0.04}^{+0.09+0.04} \times 10^{-3}$ & $2.08_{-0.01-0.06}^{+0.04+0.08} \times 10^{-3}$ & $2.16_{-0.01-0.05}^{+0.04+0.05} \times 10^{-3}$ \\
\hline & $\tilde{q} \tilde{q}$ & $9.82_{-0.67_{-0.13}^{+0.91+0.12} \times 10^{-3}}$ & $1.05_{-0.07-0.05}^{+0.05+0.05} \times 10^{-2}$ & $1.06_{-0.02-0.06}^{+0.03+0.06} \times 10^{-2}$ & $1.06_{-0.03-0.06}^{+0.04+0.05} \times 10^{-2}$ \\
\hline & $\tilde{q} \tilde{g}$ & $5.86_{-0.05-0.34}^{+0.19+0.19} \times 10^{-3}$ & $6.07_{-0.15-0.04}^{+0.00+0.04} \times 10^{-3}$ & $6.23_{-0.35-0.08}^{+0.33+0.05} \times 10^{-3}$ & $6.34_{-0.38-0.08}^{+0.36+0.04} \times 10^{-3}$ \\
\hline & $\tilde{g} \tilde{g}$ & $4.33_{-0.27-0.37}^{+0.38+0.36} \times 10^{-4}$ & $4.14_{-0.16-0.23}^{+0.00+0.23} \times 10^{-4}$ & $4.35_{-0.38-0.23}^{+0.35+0.23} \times 10^{-4}$ & $4.71_{-0.48-0.24}^{+0.43+0.25} \times 10^{-4}$ \\
\hline \multirow[t]{4}{*}{$2 ., 1.8$} & $\tilde{q} \overline{\tilde{q}}$ & $6.62_{-0.07-0.27}^{+0.28+0.31} \times 10^{-4}$ & $7.15_{-0.53-0.20}^{+0.35+0.20} \times 10^{-4}$ & $7.39_{-0.04-0.24}^{+0.11+0.31} \times 10^{-4}$ & $7.69_{-0.04-0.22}^{+0.14+0.23} \times 10^{-4}$ \\
\hline & $\tilde{q} \tilde{q}$ & $4.78_{-0.39-0.07}^{+0.53+0.05} \times 10^{-3}$ & $5.16_{-0.42-0.40}^{+0.32+0.40} \times 10^{-3}$ & $5.24_{-0.17-0.40}^{+0.22+0.41} \times 10^{-3}$ & $5.23_{-0.21-0.40}^{+0.28+0.40} \times 10^{-3}$ \\
\hline & $\tilde{q} \tilde{g}$ & $6.82_{-0.01-0.41}^{+0.17+0.22} \times 10^{-3}$ & $7.20_{-0.26-0.13}^{+0.02+0.13} \times 10^{-3}$ & $7.48_{-0.39-0.17}^{+0.42+0.15} \times 10^{-3}$ & $7.62_{-0.41-0.17}^{+0.44+0.13} \times 10^{-3}$ \\
\hline & $\tilde{g} \tilde{g}$ & $1.40_{-0.03-0.11}^{+0.08+0.11} \times 10^{-3}$ & $1.41_{-0.08-0.04}^{+0.01+0.04} \times 10^{-3}$ & $1.47_{-0.08-0.05}^{+0.09+0.05} \times 10^{-3}$ & $1.60_{-0.12-0.05}^{+0.12+0.05} \times 10^{-3}$ \\
\hline \multirow[t]{4}{*}{$2.3,2.5$} & $\tilde{q} \overline{\tilde{q}}$ & $1.32_{-0.01-0.06}^{+0.06+0.07} \times 10^{-4}$ & $1.41_{-0.12-0.03}^{+0.09+0.03} \times 10^{-4}$ & $1.48_{-0.01-0.04}^{+0.02+0.06} \times 10^{-4}$ & $1.55_{-0.01-0.03}^{+0.03+0.04} \times 10^{-4}$ \\
\hline & $\tilde{q} \tilde{q}$ & $1.21_{-0.09-0.02}^{+0.12+0.02} \times 10^{-3}$ & $1.31_{-0.10-0.07}^{+0.07+0.07} \times 10^{-3}$ & $1.33_{-0.03-0.07}^{+0.04+0.07} \times 10^{-3}$ & $1.33_{-0.04-0.07}^{+0.05+0.07} \times 10^{-3}$ \\
\hline & $\tilde{q} \tilde{g}$ & $5.13_{-0.08-0.36}^{+0.23+0.19} \times 10^{-4}$ & $5.23_{-0.19-0.03}^{+0.00+0.03} \times 10^{-4}$ & $5.49_{-0.35-0.09}^{+0.34+0.04} \times 10^{-4}$ & $5.60_{-0.38-0.09}^{+0.37+0.04} \times 10^{-4}$ \\
\hline & $\tilde{g} \tilde{g}$ & $2.84_{-0.24-0.27}^{+0.32+0.26} \times 10^{-5}$ & $2.59_{-0.14-0.12}^{+0.01+0.12} \times 10^{-5}$ & $2.82_{-0.29-0.13}^{+0.28+0.13} \times 10^{-5}$ & $3.11_{-0.36-0.14}^{+0.35+0.14} \times 10^{-5}$ \\
\hline \multirow[t]{4}{*}{$2.5,2.3$} & $\tilde{q} \overline{\tilde{q}}$ & $4.72_{-0.03-0.22}^{+0.17+0.25} \times 10^{-5}$ & $5.03_{-0.42-0.12}^{+0.31+0.12} \times 10^{-5}$ & $5.29_{-0.04-0.17}^{+0.09+0.22} \times 10^{-5}$ & $-0.16 \times 10^{-5}$ \\
\hline & $\tilde{q} \tilde{q}$ & $5.74_{-0.47-0.11}^{+0.65+0.08} \times 10^{-4}$ & $6.22_{-0.55-0.44}^{+0.44+0.44} \times 10^{-4}$ & $6.38_{-0.20-0.45}^{+0.27+0.46} \times 10^{-4}$ & $6.36_{-0.24-0.45}^{+0.34+0.44} \times 10^{-4}$ \\
\hline & $\tilde{q} \tilde{g}$ & $5.83_{-0.04-0.41}^{+0.22+0.22} \times 10^{-4}$ & $6.05_{-0.28-0.09}^{+0.05+0.09} \times 10^{-4}$ & $6.42_{-0.38-0.15}^{+0.41+0.12} \times 10^{-4}$ & $6.56_{-0.40-0.15}^{+0.44+0.09} \times 10^{-4}$ \\
\hline & $\tilde{g} \tilde{g}$ & $8.62_{-0.39-0.76}^{+0.72+0.74} \times 10^{-5}$ & $8.38_{-0.56-0.24}^{+0.19+0.24} \times 10^{-5}$ & $9.07_{-0.65-0.27}^{+0.71+0.30} \times 10^{-5}$ & $1.00_{-0.09-0.03}^{+0.09+0.03} \times 10^{-4}$ \\
\hline \multirow[t]{4}{*}{$2.8,3$} & $\tilde{q} \tilde{\tilde{q}}$ & $9.69_{-0.03-0.47}^{+0.32+0.54} \times 10^{-6}$ & $1.02_{-0.09-0.02}^{+0.07+0.02} \times 10^{-5}$ & $1.09_{-0.01-0.03}^{+0.02+0.04} \times 10^{-5}$ & $1.15_{-0.01-0.02}^{+0.03+0.03} \times 10^{-5}$ \\
\hline & $\tilde{q} \tilde{q}$ & $1.47_{-0.11-0.04}^{+0.15+0.02} \times 10^{-4}$ & $1.59_{-0.14-0.08}^{+0.10+0.08} \times 10^{-4}$ & $1.64_{-0.04-0.08}^{+0.05+0.09} \times 10^{-4}$ & $1.64_{-0.05-0.08}^{+0.07+0.08} \times 10^{-4}$ \\
\hline & $\tilde{q} \tilde{g}$ & $4.62_{-0.14-0.38}^{+0.25+0.20} \times 10^{-5}$ & $4.61_{-0.24-0.03}^{+0.03+0.03} \times 10^{-5}$ & $4.97_{-0.36-0.10}^{+0.34+0.04} \times 10^{-5}$ & $5.10_{-0.39-0.10}^{+0.37+0.03} \times 10^{-5}$ \\
\hline & $\tilde{g} \tilde{g}$ & $1.97_{-0.22-0.22}^{+0.26+0.20} \times 10^{-6}$ & $1.68_{-0.13-0.08}^{+0.02+0.08} \times 10^{-6}$ & $1.93_{-0.23-0.09}^{+0.22+0.08} \times 10^{-6}$ & $2.18_{-0.30-0.09}^{+0.27+0.10} \times 10^{-6}$ \\
\hline \multirow[t]{4}{*}{$3 ., 2.8$} & $\tilde{q} \overline{\tilde{q}}$ & $3.50_{-0.01-0.17}^{+0.09+0.20} \times 10^{-6}$ & $3.68_{-0.34-0.08}^{+0.25+0.08} \times 10^{-6}$ & $3.93_{-0.05-0.12}^{+0.08+0.17} \times 10^{-6}$ & $4.17_{-0.07-0.10}^{+0.10+0.11} \times 10^{-6}$ \\
\hline & $\tilde{q} \tilde{q}$ & $6.61_{-0.54-0.16}^{+0.76+0.11} \times 10^{-5}$ & $7.19_{-0.70-0.47}^{+0.58+0.47} \times 10^{-5}$ & $7.45_{-0.23-0.48}^{+0.31+0.50} \times 10^{-5}$ & $7.43_{-0.28-0.48}^{+0.39+0.48} \times 10^{-5}$ \\
\hline & $\tilde{q} \tilde{g}$ & $5.16_{-0.09-0.42}^{+0.25+0.23} \times 10^{-5}$ & $5.24_{-0.32-0.07}^{+0.07+0.07} \times 10^{-5}$ & $5.71_{-0.39-0.14}^{+0.40+0.10} \times 10^{-5}$ & $5.87_{-0.42-0.14}^{+0.43+0.07} \times 10^{-5}$ \\
\hline & $\tilde{g} \tilde{g}$ & $5.90_{-0.42-0.60}^{+0.63+0.57} \times 10^{-6}$ & $5.43_{-0.46-0.16}^{+0.20+0.16} \times 10^{-6}$ & $6.16_{-0.56-0.19}^{+0.58+0.21} \times 10^{-6}$ & $6.94_{-0.73-0.20}^{+0.75+0.21} \times 10^{-6}$ \\
\hline
\end{tabular}

Table 7. Different higher-order approximations for the LHC with $\sqrt{s}=13 \mathrm{TeV}$. The first error denotes the scale variation while the second error refers to the estimate of the remaining theoretical uncertainty. The latter is given by the resummation uncertainty (NLL), the variation of the two-loop constant $\left(\mathrm{NNLO}_{\mathrm{app}}\right)$, while for the two NNLL results the two errors are added in quadrature. 
for heavy sparticles. The higher-order Coulomb corrections and bound-state effects only included in the full NNLL results become important in particular for squark-antisquark and gluino-pair production at high masses, whereas the corrections are moderate for squarkgluino production and small for squark-squark production. The uncertainty from resummation ambiguities and missing higher-order corrections is strongly reduced from NLL to NNLL for all processes with the exception of squark-squark production. It is seen that this uncertainty is dominated by the two-loop constant variation, which is identical for $\mathrm{NNLO}_{\text {app }}$ and the two NNLL implementations. The scale uncertainty alone is usually reduced for the $\mathrm{NNLO}_{\text {app }}$ approximation, but can be very asymmetric as seen already in figure 6. For a more realistic uncertainty estimate at this order, the renormalization scale should be varied independently. The scale uncertainty is further reduced at NNLL for squark-antisquark and squark-squark production but increased for squark-gluino and gluino-pair production, consistent with figure 5 .

\section{Conclusions}

We performed a combined NNLL resummation of soft-gluon and Coulomb corrections for all squark- and gluino-pair production channels at the LHC based on the method developed for top-quark pair production [51], extending an earlier NLL study [16]. Grids with our NNLL predictions for the LHC with $\sqrt{s}=13$ and $14 \mathrm{TeV}$ for $m_{\tilde{q}}, m_{\tilde{g}}=200-3000 \mathrm{GeV}$ and 200 $3500 \mathrm{GeV}$, respectively, are publicly available [1]. We furthermore completed the result for the NNLO threshold expansion of the total cross section [53] by deriving the spin-dependent non-Coulomb corrections and the process-specific annihilation contributions, which both give rise to a single-logarithmic NNLO correction.

Our NNLL results show generally moderate corrections to the NLL predictions with combined soft-Coulomb corrections [16], which shows that the combined resummation is the adequate method to control the QCD corrections in the region of large sparticle masses, where both the NLO SQCD and the NLL soft-gluon and Coulomb corrections can become very large, especially for gluino-pair production. Corrections beyond NNLO included in the resummed results become sizeable for sparticle masses above $1.5 \mathrm{TeV}$. We carefully estimated uncertainties due to scale choices and ambiguities of the resummation formalism and found that the total theoretical uncertainty of the squark and gluino pair production processes due to missing higher-order corrections is reduced to the $10 \%$ level. We also compared different scale-setting procedures for the soft scale in the momentum-space formalism for soft-gluon resummation and found a better agreement compared to the NLL calculation. The NNLL calculation leaves the PDF uncertainties as the dominant source of uncertainties, which can hopefully be reduced in the future using constraints from measurements at the LHC.

Note added. In the final stages of this work we became aware of related work on the combined soft-Coulomb resummation in the Mellin-space formalism [83], where a detailed comparison to our results from [19] is performed. 


\section{Acknowledgments}

We would like to thank Pietro Falgari for collaboration in the early stages of this work, Anna Kulesza for correspondence on the hard matching coefficients computed in [59] and Michael Krämer for sharing a draft of [83]. The work of MB is supported by the BMBF grant 05H15WOCAA. The work of CW was partially funded by Research Funding Program ARISTEIA, HOCTools (co-financed by the European Union (European Social Fund ESF) and Greek national funds through the Operational Program "Education and Lifelong Learning" of the National Strategic Reference Framework (NSRF)). CS is supported by the Heisenberg Programme of the DFG. JP and CS acknowledge support by the Munich Institute for Astro- and Particle Physics (MIAPP) of the DFG cluster of excellence "Origin and Structure of the Universe" and the Mainz Institute for Theoretical Physics (MITP) during parts of this work.

\section{A Explicit formulae}

\section{A.1 Expansion of the NNLL cross section}

In this appendix we collect the expansions of the NNLL correction factors to $\mathcal{O}\left(\alpha_{s}\right)$ and $\mathcal{O}\left(\alpha_{s}^{2}\right)$, respectively. The expansion to NLO accuracy yields all threshold-enhanced NLO terms and the constant term,

$$
\begin{aligned}
f_{p p^{\prime}, i}^{\mathrm{NNL}(1)}= & -\frac{2 \pi^{2} D_{R_{\alpha}}}{\beta} \sqrt{\frac{2 m_{r}}{M}}+4 C_{r r^{\prime}}\left[\mathrm{L}_{E}^{2}+6 \ln 2 \mathrm{~L}_{E}\right] \\
& -4\left(C_{R_{\alpha}}+4 C_{r r^{\prime}}\right) \mathrm{L}_{E}+C_{p p^{\prime}, i}^{(1)}(\mu)+\mathcal{O}(\beta) .
\end{aligned}
$$

Here and in the following we use the notation

$$
\mathrm{L}_{x}=\ln \left(\frac{x}{\mu_{f}}\right),
$$

and the sum of the two quadratic Casimir operators of the colour representation of the incoming partons has been defined as

$$
C_{r r^{\prime}}=C_{r}+C_{r^{\prime}} .
$$

The constant term can be expressed in terms of the one-loop hard coefficient in (2.12) by the relation

$$
C_{p p^{\prime}, i}^{(1)}(\mu)=h_{i}^{(1)}(\mu)+4 C_{r r^{\prime}}\left[9 \ln ^{2} 2-12 \ln 2+8-\frac{11 \pi^{2}}{24}\right]-12 C_{R}[\ln 2-1] .
$$

The expansion to NNLO accuracy reads

$$
\begin{aligned}
f_{p p^{\prime}, i}^{\mathrm{NNL}(2)}= & \frac{4 \pi^{4} D_{R_{\alpha}}^{2}}{3 \beta^{2}} \frac{2 m_{\mathrm{red}}}{M}+\frac{2 \pi^{2} D_{R_{\alpha}}}{\beta} \sqrt{\frac{2 m_{\mathrm{red}}}{M}}\left\{-4 C_{r r^{\prime}}\left(\mathrm{L}_{2 E}^{2}+\mathrm{L}_{\mu_{h}}\left(\mathrm{~L}_{\mu_{h}}-2 \mathrm{~L}_{2 M}\right)-\frac{\pi^{2}}{8}\right)\right. \\
& +\mathrm{L}_{2 E}\left(\beta_{0}+4 C_{R_{\alpha}}\right)-2 \mathrm{~L}_{\mu_{h}}\left(2 C_{R_{\alpha}}+\gamma_{r r^{\prime}}^{(0)}-2 \beta_{0}\right)+\beta_{0} \mathrm{~L}_{4 m_{\mathrm{red}}} \\
& \left.-a_{1}-4 C_{R_{\alpha}}-h_{1}^{(i)}\left(\mu_{h}\right)\right\}
\end{aligned}
$$




$$
\begin{aligned}
& +8 \pi^{2} D_{R_{\alpha}}\left(C_{A}-2 D_{R_{\alpha}}\left(1+\nu_{\mathrm{spin}}^{S}\right)-\frac{1}{2} \frac{4 m_{\mathrm{red}}^{2}}{M^{2}} \nu_{\mathrm{ann}}^{R_{\alpha}, S}\right) \ln \frac{E}{M} \\
& +8 C_{r r^{\prime}}^{2}\left(\mathrm{~L}_{8 E}^{4}-\mathrm{L}_{8 E_{s}}^{4}+\mathrm{L}_{\mu_{h}}^{4}\right)-16 C_{r r^{\prime}}\left(\frac{\beta_{0}}{6}+4 C_{r r^{\prime}}+C_{R_{\alpha}}\right)\left(\mathrm{L}_{8 E}^{3}-\mathrm{L}_{8 E_{s}}^{3}\right) \\
& -8 C_{r r^{\prime}}\left[4 C_{r r^{\prime}} \mathrm{L}_{2 M}+\frac{8 \beta_{0}}{3}-\gamma_{r r^{\prime}}^{(0)}-2 C_{R_{\alpha}}\right] \mathrm{L}_{\mu_{h}}^{3} \\
& +\left\{C_{r r^{\prime}}^{2}\left(384-\frac{70 \pi^{2}}{3}\right)+4 C_{r r^{\prime}}\left(4 \beta_{0}+28 C_{R_{\alpha}}+\left(\frac{67}{3}-\pi^{2}-\frac{20 n_{f} T_{F}}{9}\right)\right)\right. \\
& \left.+4 C_{R_{\alpha}}\left(\beta_{0}+2 C_{R_{\alpha}}\right)\right\}\left(\mathrm{L}_{8 E}^{2}-\mathrm{L}_{8 E_{s}}^{2}\right) \\
& +\left\{16 C_{r r^{\prime}}^{2}\left(\mathrm{~L}_{8 E}^{2}-4 \mathrm{~L}_{8 E}+2 \mathrm{~L}_{2 M}^{2}-\frac{11 \pi^{2}}{24}+8\right)\right. \\
& +C_{r r^{\prime}}\left[-16 C_{R_{\alpha}} \mathrm{L}_{8 E}+48 C_{R_{\alpha}}-\frac{80 n_{f} T_{F}}{9}-4 \pi^{2}+\frac{268}{3}\right. \\
& \left.+\left(40 \beta_{0}-16 \gamma_{r r^{\prime}}^{(0)}-32 C_{R_{\alpha}}\right) \mathrm{L}_{2 M}\right] \\
& \left.+12 \beta_{0}^{2}-10 \beta_{0}\left(\gamma_{r r^{\prime}}^{(0)}+2 C_{R_{\alpha}}\right)+2\left(\gamma_{r r^{\prime}}^{(0)}+2 C_{R_{\alpha}}\right)^{2}\right\} \mathrm{L}_{\mu_{h}}^{2} \\
& +\left\{C_{r r^{\prime}}^{2}\left(448 \zeta(3)-1536+\frac{280 \pi^{2}}{3}\right)+C_{r r^{\prime}}\left[\left(\frac{11 \pi^{2}}{3}-64\right) \beta_{0}\right.\right. \\
& \left.+\left(\frac{70 \pi^{2}}{3}-448\right) C_{R_{\alpha}}+84 \zeta(3)+\frac{59 \pi^{2}}{3}-\frac{4024}{9}+n_{f} T_{F}\left(\frac{1184}{27}-\frac{4 \pi^{2}}{9}\right)\right] \\
& \left.+C_{R_{\alpha}}\left(-24 \beta_{0}-24 \zeta(3)+4 \pi^{2}-\frac{588-80 n_{f} T_{F}}{9}\right)-48 C_{R_{\alpha}}^{2}\right\}\left(\mathrm{L}_{8 E}-\mathrm{L}_{8 E_{s}}\right) \\
& +\left\{4 C_{r r^{\prime}}\left(\mathrm{L}_{8 E}^{2}-4 \mathrm{~L}_{8 E}+\mathrm{L}_{\mu_{h}}^{2}-\frac{11 \pi^{2}}{24}+8\right)\right. \\
& \left.-4 C_{R_{\alpha}}\left(\mathrm{L}_{8 E}-3\right)-2 \mathrm{~L}_{\mu_{h}}\left[4 C_{r r^{\prime}} \mathrm{L}_{2 M}-2 C_{R_{\alpha}}+3 \beta_{0}-\gamma_{r r^{\prime}}^{(0)}\right]\right\} h_{1}^{(i)}\left(\mu_{h}\right) \\
& +\left\{( 4 C _ { r r ^ { \prime } } \mathrm { L } _ { 2 M } + 2 \beta _ { 0 } - \gamma _ { r r ^ { \prime } } ^ { ( 0 ) } - 2 C _ { R _ { \alpha } } ) \left(-8 C_{r r^{\prime}} \mathrm{L}_{8 E}^{2}+8\left(4 C_{r r^{\prime}}+C_{R_{\alpha}}\right) \mathrm{L}_{8 E}\right.\right. \\
& \left.-64 C_{r r^{\prime}}+\frac{11 \pi^{2} C_{r r^{\prime}}}{3}-24 C_{R_{\alpha}}\right)-C_{r r^{\prime}}\left(\frac{536}{3}-\frac{160 n_{f} T_{F}}{9}-8 \pi^{2}\right) \mathrm{L}_{2 M} \\
& +C_{r r^{\prime}}\left(\frac{808}{9}-\frac{11 \pi^{2}}{3}-84 \zeta(3)-\left(\frac{224}{27}-\frac{4 \pi^{2}}{9}\right) n_{f} T_{F}\right) \\
& \left.+2 \gamma_{r r^{\prime}}^{(1)}+C_{R_{\alpha}}\left(24 \zeta(3)+\frac{588-80 n_{f} T_{F}}{9}-4 \pi^{2}\right)-4 \beta_{1}\right\} \mathrm{L}_{\mu_{h}}+C_{p p^{\prime}, i}^{(2)} .
\end{aligned}
$$

Here we further defined $\mathrm{L}_{8 E_{s}}=\ln \left(\frac{8 E}{\mu_{s}}\right)$. The two-loop beta-function coefficient is given by $\beta_{1}=\frac{34}{3} C_{A}^{2}-\frac{20}{3} C_{A} T_{F} n_{f}-4 C_{F} T_{F} n_{f}$. The anomalous-dimension coefficients appearing 
in this formula are related to the incoming partons and are defined as

$$
\gamma_{r r^{\prime}}^{(n)}=\gamma^{\phi, r(n)}+\gamma^{\phi, r^{\prime}(n)}
$$

with

$$
\begin{aligned}
\gamma^{\phi, 3(0)}= & 3 C_{F} \\
\gamma^{\phi, 3(1)}= & C_{F}^{2}\left(\frac{3}{2}-2 \pi^{2}+24 \zeta_{3}\right)+C_{A} C_{F}\left(\frac{17}{6}+\frac{22 \pi^{2}}{9}-12 \zeta_{3}\right) \\
& -C_{F} T_{F} n_{f}\left(\frac{2}{3}+\frac{8 \pi^{2}}{9}\right), \\
\gamma^{\phi, 8(0)}= & \beta_{0}=\frac{11}{3} C_{A}-\frac{4}{3} T_{F} n_{f}, \\
\gamma^{\phi, 8(1)}= & 4 C_{A}^{2}\left(\frac{8}{3}+3 \zeta_{3}\right)-\frac{16}{3} C_{A} T_{F} n_{f}-4 C_{F} T_{F} n_{f} .
\end{aligned}
$$

\section{A.2 Analytic NNLL result for fixed-order Coulomb corrections}

If the Coulomb corrections are treated at fixed NNLO accuracy through the factor (2.52), the $\omega$-convolution in the resummed cross section (2.10) can be performed explicitly, resulting in an analytic expression:

$$
\hat{\sigma}_{p p^{\prime}}^{\mathrm{res}}(\hat{s}, \mu)=\sum_{i} \sigma_{p p^{\prime}, i}^{(0)}(\mu) U_{R_{\alpha}}\left(\mu_{h}, \mu_{s}, \mu_{f}\right)\left(\frac{2 M}{\mu_{s}}\right)^{-2 \eta} \tilde{s}_{i}^{R_{\alpha}}\left(\partial_{\eta}, \mu_{s}\right) \mathcal{C}_{\mathrm{hC}}^{\mathrm{NNLO}}\left(E, \mu_{h}, \mu_{s}, \mu_{f}\right)
$$

with the Laplace transform of the NLO soft function (2.11) and where the function $\mathcal{C}_{\mathrm{hC}}^{\mathrm{NNO}}$ is given by

$$
\begin{aligned}
\mathcal{C}_{\mathrm{hC}}^{\mathrm{NNLO}}\left(E, \mu_{h}, \mu_{s}, \mu_{f}\right) & =\left(\frac{2 E e^{-\gamma_{E}}}{\mu_{s}}\right)^{2 \eta} \sum_{n=0}^{2}\left(\frac{\alpha_{s}}{4 \pi}\right)^{n} \mathcal{C}_{\mathrm{hC}}^{(n)}\left(E, \mu_{h}, \mu_{s}, \mu_{f}\right), \\
\mathcal{C}_{\mathrm{hC}}^{(0)}\left(E, \mu_{h}, \mu_{s}, \mu_{f}\right) & =\frac{\sqrt{\pi}}{2 \Gamma\left(2 \eta+\frac{3}{2}\right)}, \\
\mathcal{C}_{\mathrm{hC}}^{(1)}\left(E, \mu_{h}, \mu_{s}, \mu_{f}\right)= & -\frac{\left(2 \pi^{2} D_{R_{\alpha}}\right)}{\Gamma(2 \eta+1)} \sqrt{\frac{2 m_{\mathrm{red}}}{E}}+\frac{\sqrt{\pi}}{2 \Gamma\left(2 \eta+\frac{3}{2}\right)} h_{i}^{(1)}\left(\mu_{h}\right), \\
\mathcal{C}_{\mathrm{hC}}^{(2)}\left(E, \mu_{h}, \mu_{s}, \mu_{f}\right)= & \frac{\sqrt{\pi}\left(2 \pi^{2} D_{R_{\alpha}}\right)^{2}}{3 \Gamma\left(2 \eta+\frac{1}{2}\right)}\left(\frac{2 m_{\mathrm{red}}}{E}\right)-\frac{\left(2 \pi^{2} D_{R_{\alpha}}\right)}{\Gamma(2 \eta+1)} \sqrt{\frac{2 m_{\mathrm{red}}}{E}} \\
& \times\left[h_{1}^{(i)}\left(\mu_{h}\right)+a_{1}-\beta_{0}\left(\ln \left(\frac{8 E m_{\mathrm{red}}}{\mu^{2}}\right)-\psi^{(0)}(2 \eta+1)-\gamma_{E}\right)\right] \\
& +\frac{2 \sqrt{\pi}\left(2 \pi^{2} D_{R_{\alpha}}\right)}{\Gamma\left(2 \eta+\frac{3}{2}\right)}\left(C_{A}-2 D_{R_{\alpha}}\left(v_{\mathrm{spin}}+1\right)-\frac{\nu_{\mathrm{ann}}, S}{2} \frac{4 m_{\mathrm{red}}^{2}}{M^{2}}\right) \\
& \times\left(\ln \left(\frac{E}{M}\right)-\psi^{(0)}\left(2 \eta+\frac{3}{2}\right)-\gamma_{E}+2-2 \ln 2\right) \\
& +\frac{\sqrt{\pi}}{2 \Gamma\left(2 \eta+\frac{3}{2}\right)} h_{i}^{(1)}\left(\mu_{h}\right)\left(-2 \beta_{0}\right) \ln \left(\frac{\mu_{h}}{\mu_{f}}\right)
\end{aligned}
$$

with the digamma function $\psi^{(0)}(x)=\frac{d \ln \Gamma(x)}{d x}$. It is straightforward to evaluate the action of the derivative with respect to $\eta$ in (A.11) on the factors (A.13)-(A.15). 
Open Access. This article is distributed under the terms of the Creative Commons Attribution License (CC-BY 4.0), which permits any use, distribution and reproduction in any medium, provided the original author(s) and source are credited.

\section{References}

[1] M. Beneke, J. Piclum, C. Schwinn and C. Wever, SUSYNNLL, http://users.ph.tum.de/t31software/SUSYNNLL/.

[2] ATLAS collaboration, Search for squarks and gluinos with the ATLAS detector in final states with jets and missing transverse momentum using $\sqrt{s}=8$ TeV proton-proton collision data, JHEP 09 (2014) 176 [arXiv:1405.7875] [INSPIRE].

[3] CMS collaboration, Searches for Supersymmetry using the $M_{T 2}$ Variable in Hadronic Events Produced in pp Collisions at $8 \mathrm{TeV}$, JHEP 05 (2015) 078 [arXiv: 1502.04358] [INSPIRE].

[4] CMS collaboration, Search for new physics with the $M_{T 2}$ variable in all-jets final states produced in pp collisions at $\sqrt{s}=13 \mathrm{TeV}$, arXiv:1603.04053 [INSPIRE].

[5] W. Beenakker, R. Höpker, M. Spira and P.M. Zerwas, Squark and gluino production at hadron colliders, Nucl. Phys. B 492 (1997) 51 [hep-ph/9610490] [INSPIRE].

[6] W. Beenakker, R. Höpker and M. Spira, PROSPINO: A Program for the production of supersymmetric particles in next-to-leading order QCD, hep-ph/9611232 [INSPIRE].

[7] A. Kulesza and L. Motyka, Threshold resummation for squark-antisquark and gluino-pair production at the LHC, Phys. Rev. Lett. 102 (2009) 111802 [arXiv:0807.2405] [INSPIRE].

[8] A. Kulesza and L. Motyka, Soft gluon resummation for the production of gluino-gluino and squark-antisquark pairs at the LHC, Phys. Rev. D 80 (2009) 095004 [arXiv:0905.4749] [INSPIRE].

[9] U. Langenfeld and S.-O. Moch, Higher-order soft corrections to squark hadro-production, Phys. Lett. B 675 (2009) 210 [arXiv:0901.0802] [INSPIRE].

[10] K. Hagiwara and H. Yokoya, Bound-state effects on gluino-pair production at hadron colliders, JHEP 10 (2009) 049 [arXiv:0909.3204] [INSPIRE].

[11] W. Beenakker et al., Soft-gluon resummation for squark and gluino hadroproduction, JHEP 12 (2009) 041 [arXiv: 0909.4418].

[12] M. Beneke, P. Falgari and C. Schwinn, Threshold resummation for pair production of coloured heavy (s)particles at hadron colliders, Nucl. Phys. B 842 (2011) 414 [arXiv: 1007.5414] [INSPIRE].

[13] M.R. Kauth, J.H. Kühn, P. Marquard and M. Steinhauser, Gluino Pair Production at the LHC: The Threshold, Nucl. Phys. B 857 (2012) 28 [arXiv:1108.0361] [InSPIRE].

[14] M.R. Kauth, A. Kress and J.H. Kühn, Gluino-Squark Production at the LHC: The Threshold, JHEP 12 (2011) 104 [arXiv: 1108.0542] [INSPIRE].

[15] W. Beenakker, S. Brensing, M. Krämer, A. Kulesza, E. Laenen and I. Niessen, NNLL resummation for squark-antisquark pair production at the LHC, JHEP 01 (2012) 076 [arXiv: 1110.2446$]$ [INSPIRE].

[16] P. Falgari, C. Schwinn and C. Wever, NLL soft and Coulomb resummation for squark and gluino production at the LHC, JHEP 06 (2012) 052 [arXiv:1202.2260] [INSPIRE]. 
[17] U. Langenfeld, S.-O. Moch and T. Pfoh, QCD threshold corrections for gluino pair production at hadron colliders, JHEP 11 (2012) 070 [arXiv: 1208.4281] [INSPIRE].

[18] T. Pfoh, Phenomenology of QCD threshold resummation for gluino pair production at NNLL, JHEP 05 (2013) 044 [Erratum ibid. 10 (2013) 090] [arXiv: 1302.7202] [INSPIRE].

[19] M. Beneke, P. Falgari, J. Piclum, C. Schwinn and C. Wever, Higher-order soft and Coulomb corrections to squark and gluino production at the LHC, PoS(RADCOR 2013) 051 [arXiv: 1312.0837] [INSPIRE].

[20] W. Beenakker, C. Borschensky, M. Krämer et al., NNLL resummation for squark and gluino production at the LHC, JHEP 12 (2014) 023 [arXiv:1404.3134] [INSPIRE].

[21] W. Beenakker, M. Krämer, T. Plehn, M. Spira and P.M. Zerwas, Stop production at hadron colliders, Nucl. Phys. B 515 (1998) 3 [hep-ph/9710451] [INSPIRE].

[22] J.E. Younkin and S.P. Martin, QCD corrections to stoponium production at hadron colliders, Phys. Rev. D 81 (2010) 055006 [arXiv:0912.4813] [inSPIRE].

[23] W. Beenakker, S. Brensing, M. Krämer, A. Kulesza, E. Laenen and I. Niessen, Supersymmetric top and bottom squark production at hadron colliders, JHEP 08 (2010) 098 [arXiv: 1006.4771] [INSPIRE].

[24] U. Langenfeld, Threshold Improved QCD Corrections for Stop-Antistop production at Hadron colliders, JHEP 07 (2011) 052 [arXiv: 1011.3341] [INSPIRE].

[25] A. Broggio, A. Ferroglia, M. Neubert, L. Vernazza and L.L. Yang, Approximate NNLO Predictions for the Stop-Pair Production Cross Section at the LHC, JHEP 07 (2013) 042 [arXiv: 1304.2411] [INSPIRE].

[26] A. Broggio, A. Ferroglia, M. Neubert, L. Vernazza and L.L. Yang, NNLL Momentum-Space Resummation for Stop-Pair Production at the LHC, JHEP 03 (2014) 066 [arXiv: 1312.4540] [INSPIRE].

[27] C. Kim, A. Idilbi, T. Mehen and Y.W. Yoon, Production of Stoponium at the LHC, Phys. Rev. D 89 (2014) 075010 [arXiv:1401.1284] [INSPIRE].

[28] W. Beenakker, C. Borschensky, R. Heger, M. Krämer, A. Kulesza and E. Laenen, NNLL resummation for stop pair-production at the LHC, JHEP 05 (2016) 153 [arXiv:1601.02954] [INSPIRE].

[29] S. Bornhauser, M. Drees, H.K. Dreiner and J.S. Kim, Electroweak contributions to squark pair production at the LHC, Phys. Rev. D 76 (2007) 095020 [arXiv:0709.2544] [INSPIRE].

[30] W. Hollik, M. Kollar and M.K. Trenkel, Hadronic production of top-squark pairs with electroweak NLO contributions, JHEP 02 (2008) 018 [arXiv:0712.0287] [INSPIRE].

[31] W. Hollik and E. Mirabella, Squark anti-squark pair production at the LHC: The Electroweak contribution, JHEP 12 (2008) 087 [arXiv:0806.1433] [INSPIRE].

[32] W. Hollik, E. Mirabella and M.K. Trenkel, Electroweak contributions to squark-gluino production at the LHC, JHEP 02 (2009) 002 [arXiv:0810.1044] [INSPIRE].

[33] E. Mirabella, NLO electroweak contributions to gluino pair production at hadron colliders, JHEP 12 (2009) 012 [arXiv:0908.3318] [INSPIRE].

[34] J. Germer, W. Hollik, E. Mirabella and M.K. Trenkel, Hadronic production of squark-squark pairs: The electroweak contributions, JHEP 08 (2010) 023 [arXiv: 1004.2621] [INSPIRE]. 
[35] J. Germer, W. Hollik, J.M. Lindert and E. Mirabella, Top-squark pair production at the LHC: a complete analysis at next-to-leading order, JHEP 09 (2014) 022 [arXiv:1404.5572] [INSPIRE].

[36] W. Hollik, J.M. Lindert, E. Mirabella and D. Pagani, Electroweak corrections to squark-antisquark production at the LHC, JHEP 08 (2015) 099 [arXiv:1506.01052] [INSPIRE].

[37] D. Gonçalves-Netto, D. López-Val, K. Mawatari, T. Plehn and I. Wigmore, Automated Squark and Gluino Production to Next-to-Leading Order, Phys. Rev. D 87 (2013) 014002 [arXiv: 1211.0286] [INSPIRE].

[38] D. Goncalves, D. Lopez-Val, K. Mawatari and T. Plehn, Automated third generation squark production to next-to-leading order, Phys. Rev. D 90 (2014) 075007 [arXiv:1407.4302] [INSPIRE].

[39] R. Gavin, C. Hangst, M. Krämer et al., Matching Squark Pair Production at NLO with Parton Showers, JHEP 10 (2013) 187 [arXiv:1305.4061] [INSPIRE].

[40] R. Gavin, C. Hangst, M. Krämer et al., Squark Production and Decay matched with Parton Showers at NLO, Eur. Phys. J. C 75 (2015) 29 [arXiv:1407.7971] [InSPIRE].

[41] C. Degrande, B. Fuks, V. Hirschi, J. Proudom and H.-S. Shao, Matching next-to-leading order predictions to parton showers in supersymmetric QCD, Phys. Lett. B $\mathbf{7 5 5}$ (2016) 82 [arXiv: 1510.00391] [INSPIRE].

[42] W. Hollik, J.M. Lindert and D. Pagani, NLO corrections to squark-squark production and decay at the LHC, JHEP 03 (2013) 139 [arXiv:1207.1071] [INSPIRE].

[43] W. Hollik, J.M. Lindert and D. Pagani, On cascade decays of squarks at the LHC in NLO QCD, Eur. Phys. J. C 73 (2013) 2410 [arXiv:1303.0186] [InSPIRE].

[44] P. Falgari, C. Schwinn and C. Wever, Finite-width effects on threshold corrections to squark and gluino production, JHEP 01 (2013) 085 [arXiv: 1211.3408] [INSPIRE].

[45] G.F. Sterman, Summation of Large Corrections to Short Distance Hadronic Cross-Sections, Nucl. Phys. B 281 (1987) 310 [INSPIRE].

[46] S. Catani and L. Trentadue, Resummation of the QCD Perturbative Series for Hard Processes, Nucl. Phys. B 327 (1989) 323 [InSPIRE].

[47] N. Kidonakis and G.F. Sterman, Resummation for QCD hard scattering, Nucl. Phys. B 505 (1997) 321 [hep-ph/9705234] [INSPIRE].

[48] R. Bonciani, S. Catani, M.L. Mangano and P. Nason, NLL resummation of the heavy quark hadroproduction cross-section, Nucl. Phys. B 529 (1998) 424 [Erratum ibid. B 803 (2008) 234] [hep-ph/9801375] [INSPIRE].

[49] A.H. Hoang et al., Top-antitop pair production close to threshold: Synopsis of recent NNLO results, Eur. Phys. J. direct C 3 (2000) 1 [hep-ph/0001286] [INSPIRE].

[50] M. Beneke, P. Falgari and C. Schwinn, Soft radiation in heavy-particle pair production: All-order colour structure and two-loop anomalous dimension, Nucl. Phys. B $\mathbf{8 2 8}$ (2010) 69 [arXiv: 0907.1443] [INSPIRE].

[51] M. Beneke, P. Falgari, S. Klein and C. Schwinn, Hadronic top-quark pair production with NNLL threshold resummation, Nucl. Phys. B 855 (2012) 695 [arXiv:1109.1536] [InSPIRE]. 
[52] M. Beneke, P. Falgari, S. Klein et al., Inclusive Top-Pair Production Phenomenology with TOPIXS, JHEP 07 (2012) 194 [arXiv:1206.2454] [INSPIRE].

[53] M. Beneke, M. Czakon, P. Falgari, A. Mitov and C. Schwinn, Threshold expansion of the $g g(q \bar{q}) \rightarrow Q \bar{Q}+X$ cross section at $\mathcal{O}\left(\alpha_{s}^{4}\right)$, Phys. Lett. B $690(2010) 483$ [arXiv:0911.5166] [INSPIRE].

[54] P. Bärnreuther, M. Czakon and P. Fiedler, Virtual amplitudes and threshold behaviour of hadronic top-quark pair-production cross sections, JHEP 02 (2014) 078 [arXiv:1312.6279] [INSPIRE].

[55] G. Sterman and M. Zeng, Quantifying Comparisons of Threshold Resummations, JHEP 05 (2014) 132 [arXiv: 1312.5397] [INSPIRE].

[56] G.L. Kane and J.P. Leveille, Experimental Constraints on Gluino Masses and Supersymmetric Theories, Phys. Lett. B 112 (1982) 227 [INSPIRE].

[57] P.R. Harrison and C.H. Llewellyn Smith, Hadroproduction of Supersymmetric Particles, Nucl. Phys. B 213 (1983) 223 [Erratum ibid. B 223 (1983) 542] [InSPIRE].

[58] S. Dawson, E. Eichten and C. Quigg, Search for Supersymmetric Particles in Hadron-Hadron Collisions, Phys. Rev. D 31 (1985) 1581 [INSPIRE].

[59] W. Beenakker, T. Janssen, S. Lepoeter et al., Towards NNLL resummation: hard matching coefficients for squark and gluino hadroproduction, JHEP 10 (2013) 120 [arXiv:1304.6354] [INSPIRE].

[60] T. Becher and M. Neubert, Threshold resummation in momentum space from effective field theory, Phys. Rev. Lett. 97 (2006) 082001 [hep-ph/0605050] [InSPIRE].

[61] T. Becher, M. Neubert and G. Xu, Dynamical Threshold Enhancement and Resummation in Drell-Yan Production, JHEP 07 (2008) 030 [arXiv: 0710.0680] [INSPIRE].

[62] M. Beneke, Y. Kiyo and K. Schuller, Third-order correction to top-quark pair production near threshold I. Effective theory set-up and matching coefficients, arXiv:1312.4791 [INSPIRE].

[63] Y. Kats and M.D. Schwartz, Annihilation decays of bound states at the LHC, JHEP 04 (2010) 016 [arXiv:0912.0526] [INSPIRE].

[64] M. Beneke, A. Signer and V.A. Smirnov, Top quark production near threshold and the top quark mass, Phys. Lett. B 454 (1999) 137 [hep-ph/9903260] [INSPIRE].

[65] A. Pineda and A. Signer, Renormalization group improved sum rule analysis for the bottom quark mass, Phys. Rev. D 73 (2006) 111501 [hep-ph/0601185] [INSPIRE].

[66] M. Beneke, C. Hellmann and P. Ruiz-Femenia, Non-relativistic pair annihilation of nearly mass degenerate neutralinos and charginos I. General framework and S-wave annihilation, JHEP 03 (2013) 148 [Erratum ibid. 10 (2013) 224] [arXiv:1210.7928] [INSPIRE].

[67] A. Pineda and J. Soto, Matching at one loop for the four quark operators in NRQCD, Phys. Rev. D 58 (1998) 114011 [hep-ph/9802365] [INSPIRE].

[68] J. Rosiek, Complete set of Feynman rules for the minimal supersymmetric extension of the standard model, Phys. Rev. D 41 (1990) 3646 [hep-ph/9511250] [INSPIRE].

[69] A. Pineda and F.J. Yndurain, Calculation of quarkonium spectrum and $m_{b}, m_{c}$ to order $\alpha_{S}^{4}$, Phys. Rev. D 58 (1998) 094022 [hep-ph/9711287] [INSPIRE]. 
[70] M. Beneke, Y. Kiyo and K. Schuller, Third-order Coulomb corrections to the S-wave Green function, energy levels and wave functions at the origin, Nucl. Phys. B 714 (2005) 67 [hep-ph/0501289] [INSPIRE].

[71] M. Bonvini, S. Forte, G. Ridolfi and L. Rottoli, Resummation prescriptions and ambiguities in SCET vs. direct QCD: Higgs production as a case study, JHEP 01 (2015) 046 [arXiv: 1409.0864] [INSPIRE].

[72] J. Butterworth et al., PDF 4 LHC recommendations for LHC Run II, J. Phys. G 43 (2016) 023001 [arXiv: 1510.03865] [INSPIRE].

[73] L.A. Harland-Lang, A.D. Martin, P. Motylinski and R.S. Thorne, Parton distributions in the LHC era: MMHT 2014 PDFs, Eur. Phys. J. C 75 (2015) 204 [arXiv:1412.3989] [INSPIRE].

[74] S. Dulat, T.-J. Hou, J. Gao et al., New parton distribution functions from a global analysis of quantum chromodynamics, Phys. Rev. D 93 (2016) 033006 [arXiv:1506.07443] [InSPIRE].

[75] NNPDF collaboration, R.D. Ball et al., Parton distributions for the LHC Run II, JHEP 04 (2015) 040 [arXiv: 1410.8849] [INSPIRE].

[76] G. Watt and R.S. Thorne, Study of Monte Carlo approach to experimental uncertainty propagation with MSTW 2008 PDFs, JHEP 08 (2012) 052 [arXiv: 1205.4024] [INSPIRE].

[77] J. Gao and P. Nadolsky, A meta-analysis of parton distribution functions, JHEP 07 (2014) 035 [arXiv: 1401.0013] [INSPIRE].

[78] S. Carrazza, S. Forte, Z. Kassabov, J.I. Latorre and J. Rojo, An Unbiased Hessian Representation for Monte Carlo PDFs, Eur. Phys. J. C 75 (2015) 369 [arXiv:1505.06736] [INSPIRE].

[79] D.B. Clark, E. Godat and F.I. Olness, ManeParse: a Mathematica reader for Parton Distribution Functions, arXiv:1605.08012 [INSPIRE].

[80] C. Borschensky, M. Krämer, A. Kulesza et al., Squark and gluino production cross sections in pp collisions at $\sqrt{s}=13,14,33$ and $100 \mathrm{TeV}$, Eur. Phys. J. C 74 (2014) 3174 [arXiv: 1407.5066] [INSPIRE].

[81] W. Beenakker, C. Borschensky, M. Krämer et al., NLO+NLL squark and gluino production cross-sections with threshold-improved parton distributions, Eur. Phys. J. C 76 (2016) 53 [arXiv: 1510.00375] [INSPIRE].

[82] M. Bonvini, S. Marzani, J. Rojo et al., Parton distributions with threshold resummation, JHEP 09 (2015) 191 [arXiv: 1507.01006] [INSPIRE].

[83] W. Beenakker, C. Borschensky, M. Krämer, A. Kulesza and E. Laenen, NNLL-fast: predictions for coloured supersymmetric particle production at the LHC with threshold and Coulomb resummation, arXiv: 1607.07741 [INSPIRE]. 